\title{
On Sums of Generating Sets in $\mathbb{Z}_{2}^{n}$
}

\author{
Chaim Even-Zohar *
}

October 10, 2018

\begin{abstract}
Let $A$ and $B$ be two affinely generating sets of $\mathbb{Z}_{2}^{n}$. As usual, we denote their Minkowski sum by $A+B$. How small can $A+B$ be, given the cardinalities of $A$ and $B$ ? We give a tight answer to this question. Our bound is attained when both $A$ and $B$ are unions of cosets of a certain subgroup of $\mathbb{Z}_{2}^{n}$. These cosets are arranged as Hamming balls, the smaller of which has radius 1 .

By similar methods, we re-prove the Freiman-Ruzsa theorem in $\mathbb{Z}_{2}^{n}$, with an optimal upper bound. Denote by $F(K)$ the maximal spanning constant $|\langle A\rangle| /|A|$ over all subsets $A \subseteq \mathbb{Z}_{2}^{n}$ with doubling constant $|A+A| /|A| \leq K$. We explicitly calculate $F(K)$, and in particular show that $4^{K} / 4 K \leq F(K) \cdot(1+o(1)) \leq 4^{K} / 2 K$. This improves the estimate $F(K)=p o l y(K) 4^{K}$, found recently by Green and Tao [17] and by Konyagin [23.
\end{abstract}

\section{Introduction}

Much work has been devoted to the study of Minkowski sums of sets. Questions concerning such sums come up in geometry, and are at the core of additive combinatorics. Research in this area has blossomed in recent years, and even Tao and Vu's monograph [36. no longer covers all the most recent developments. In this paper we concentrate on the Minkowski sum of two generating sets of $\mathbb{Z}_{2}^{n}$.

We first review some of the relevant literature. Let $G$ be an abelian group, and let $A$ and $B$ be two finite subsets of $G$. As usual, we denote

$$
A+B=\{a+b \mid a \in A, b \in B\}
$$

and we ask about the minimum of $|A+B|$, given the cardinalities of $A$ and $B$.

In general, the answer ranges from $\max (|A|,|B|)$ to $|A|+|B|-1$, depending on the structure of $G$. For a torsion-free $G$, if $A$ and $B$ are arithmetic progressions with the same step, then $|A+B|=|A|+|B|-1$, which is optimal. Likewise, if $G=\mathbb{Z}_{p}$ is cyclic of prime order, then the answer is given by the Cauchy-Davenport theorem, $|A+B| \geq \min (|A|+|B|-1,|G|)[2$, 4. Moreover, by a theorem of Vosper 38 , if $|A|+|B|<|G|$ then equality holds only for arithmetic progressions. In the other extreme case, $G$ has a finite subgroup of a suitable cardinality. Thus, if $H \triangleleft G$ is a subgroup of cardinality $|H|=\max (|A|,|B|)$, an optimal choice is to have $A$ and $B$ be subsets of $H$, in which case $|A+B|=\max (|A|,|B|)$. More generally, $|A+B|$ can be as small as $\max (|A|,|B|)$ if and only if $\min (|A|,|B|) \leq|H|$ and $|H|$ divides $\max (|A|,|B|)[36$, p. 55]. In the general case [8, 10, the smallest possible cardinality of $|A+B|$ is $\min (\lceil|A| /|H|\rceil+\lceil|B| /|H|\rceil-1)|H|$, where the minimum is over all finite subgroups $H$ of $G$. In a sense, this result interpolates between the two extremes. In an optimal construction [1, 10, the sets $A$ and $B$ are contained in $\lceil|A| /|H|\rceil$ and $\lceil|B| /|H|\rceil$ cosets of $H$, whose arrangement is a lexicographical variant of an arithmetic progression. In particular, for $G$ a 2-torsion group this reduces to the well-studied Hopf-Stiefel function [20, 35, 39, 1, 7, 9 .

Stability is a recurring theme in modern extremal combinatorics. Once an extremal problem is solved, it is interesting to explore what happens when we consider candidate solutions that do not

\footnotetext{
*Einstein Institute of Mathematics, The Hebrew University, Jerusalem 91904, Israel, chaim.evenzohar@mail.huji.ac.il. This paper is based on the author's MSc thesis, under the supervision of Prof. Nati Linial.
} 
resemble the global optimum. The crucial feature of the above-mentioned optimal constructions is that $A$ and $B$ are densely packed in cosets of properly chosen subgroups of $G$. We therefore return to the original question, under the requirement that $A$ and $B$ are not allowed to be contained in a proper subgroup of $G$ or a coset thereof. The affine span of $A$, denoted $\langle A\rangle$, is the smallest coset (of any subgroup) containing $A$. We say that $A$ affinely generates $G$ if $\langle A\rangle=G$. Clearly this definition coincides with the usual notion of a generating set if $0 \in A$. The refined problem is as follows: In a finitely generated abelian group $G$, find $\min |A+B|$ as a function of $|A|$ and $|B|$, where $A$ and $B$ are finite affinely generating subsets of $G$.

Naturally, the structural properties of $G$ play a role in this problem as well. For the torsion-free case, $G=\mathbb{Z}^{d}$, this question and similar ones were discussed by Ruzsa 30, and a full answer was finally given by Gardner and Gronchi 15. In the extremal construction, the smaller set is a simplex of $d+1$ points, on one of whose edges lies an arithmetic progression, and the other set is roughly the sum of several copies of it. As discussed there, this is analogous to the Brunn-Minkowski theorem [33.

Here we present the following lower bound for the opposite extreme of a 2-torsion group, $G=\mathbb{Z}_{2}^{n}$.

Theorem 1. Suppose $A, B \subseteq G=\mathbb{Z}_{2}^{n}$ such that $\langle A\rangle=G, B \neq \varnothing$ and $|A| \leq \frac{3}{4}|G|$.

If $t$ is the largest positive integer such that

$$
|A| \leq \frac{t+1}{2^{t}} \cdot|G|
$$

and $0 \leq k<t$ and $w \in[-1,1]$ are such that

$$
|B|=\frac{\left(\begin{array}{l}
t \\
0
\end{array}\right)+\left(\begin{array}{l}
t \\
1
\end{array}\right)+\ldots+\left(\begin{array}{l}
t \\
k
\end{array}\right)+w\left(\begin{array}{c}
t-1 \\
k
\end{array}\right)}{2^{t}} \cdot|G|
$$

then

$$
|A+B| \geq \frac{\left(\begin{array}{l}
t \\
0
\end{array}\right)+\left(\begin{array}{l}
t \\
1
\end{array}\right)+\ldots+\left(\begin{array}{l}
t \\
k
\end{array}\right)+\left(\begin{array}{c}
t \\
k+1
\end{array}\right)+w\left(\begin{array}{l}
t-1 \\
k+1
\end{array}\right)}{2^{t}} \cdot|G|
$$

This bound is tight when $w=0$, and it is attained by the sets

$$
A=D_{1}^{t} \times \mathbb{Z}_{2}^{n-t} \quad B=D_{k}^{t} \times \mathbb{Z}_{2}^{n-t} \quad A+B=D_{k+1}^{t} \times \mathbb{Z}_{2}^{n-t}
$$

where $D_{k}^{t}=\left\{x \in \mathbb{Z}_{2}^{t} \mid \#\left\{i \mid x_{i}=1\right\} \leq k\right\}$ is a Hamming ball of radius $k$ in $\mathbb{Z}_{2}^{t}$.

The Freiman-Ruzsa theorem [31] is a major result in additive combinatorics. In the context of the above discussion, it addresses the special case $A=B$. It states that if $A$ is a subset of an $r$-torsion abelian group with $|A+A| \leq K|A|$, then $A$ is contained in a coset of cardinality at most $F(K)|A|$, with $F(K)=K^{2} r^{4}$. The special case $r=2$ has received considerable attention [5, 6, 16, 17, 19, 23, 26, 32, 37. Among the most recent contributions is work by Green and Tao [1] with further improvement by Konyagin $\left[23\right.$. It shows that one can take $F(K)=2^{2 K+O(\log K)}$. Here we exactly determine the lowest possible value of $F(K)$ for $r=2$.

Theorem 2. For $K \geq 1$, denote by $t \geq 1$ the unique integer for which:

$$
\frac{\left(\begin{array}{l}
t \\
2
\end{array}\right)+t+1}{t+1} \leq K<\frac{\left(\begin{array}{c}
t+1 \\
2
\end{array}\right)+(t+1)+1}{(t+1)+1}
$$

For $A \subseteq \mathbb{Z}_{2}^{n}$ such that $|A+A| /|A| \leq K$, we have $|\langle A\rangle| /|A| \leq F(K)$ where:

$$
F(K)= \begin{cases}\frac{2^{t}}{\left(\begin{array}{l}
t \\
2
\end{array}\right)+t+1} \cdot K & \frac{\left(\begin{array}{l}
t \\
2
\end{array}\right)+t+1}{t+1} \leq K<\frac{t^{2}+t+1}{2 t} \\
\frac{2^{t+1}}{t^{2}+t+1} \cdot K & \frac{t^{2}+t+1}{2 t} \leq K<\frac{\left(\begin{array}{c}
t+1 \\
2
\end{array}\right)+(t+1)+1}{(t+1)+1}\end{cases}
$$

This choice of $F(K)$ is tight, and grows as $\Theta\left(2^{2 K} / K\right)$.

Compression is an important tool from extremal set theory. Much progress in the application of compression to additive problems was made by Bollobás and Leader in [1, and it is a key ingredient in Green and Tao's proof in [17]. There is a whole range of compression operators $C$, that transform 
an arbitrary set $A$ to another set $C(A)$, with $|C(A)|=|A|$ and $|C(A)+C(A)| \leq|A+A|$. By a finite sequence of such compressions, it is possible to reduce to the case where $A$ is compressed in some appropriate sense, and hence has certain structural properties, which make $A+A$ easier to study. The difficulty is that $C(A)$ need not be affinely generating even if $A$ is. Green and Tao handled this difficulty by restricting the types of compression operators they used. Our approach is different. We employ more types of compression operators and we proceed as long as possible without jeopardizing affine generation, i.e., as long as $\langle C(A)\rangle=\langle A\rangle$.

Isoperimetric inequalities play an important role in our work. In our investigations of $A+B$, we prove a new variant of the isoperimetric inequality for the hypercube.

Overview: In Section 2 we discuss compressions and other useful tools. We explore the key notion of compression that maintains affine generation. In Section 3 Theorem 2 is proved, first in an asymptotic form, then with the exact expression. In Section 4 we establish Theorem 1 . The proof utilizes our new isoperimetric inequality.

\section{Tools}

In this section we briefly survey several concepts and results that are used below. These include the lexicographic order and the Hopf-Stiefel function. Then we discuss compressions in $\mathbb{Z}_{2}^{n}$, in line with Section 2 of [17, and we introduce the study of compressions that preserve affine generation.

\subsection{The Lexicographic Order}

Throughout, we use the linear basis $\left\{e_{1}, e_{2}, \ldots, e_{n}\right\}$ for $\mathbb{Z}_{2}^{n}$. Elements $x \in \mathbb{Z}_{2}^{n}$ are expressed as $x=\sum_{i=1}^{n} x_{i} e_{i}$. The correspondence between vectors $x \in \mathbb{Z}_{2}^{n}$ and their supports $\left\{j \mid x_{j}=1\right\} \subseteq$ $\{1, \ldots, n\}=[n]$, is used to simplify certain notation and arguments.

The lexicographic order is a total order on $\mathbb{Z}_{2}^{n}$. For $x, y \in \mathbb{Z}_{2}^{n}$ we say that $x \prec y$, if $x_{i}<y_{i}$ for the largest coordinate $i$ for which $x_{i} \neq y_{i}$. For example, the ordering of $\mathbb{Z}_{2}^{3}$ is:

$$
0 \prec e_{1} \prec e_{2} \prec e_{1}+e_{2} \prec e_{3} \prec e_{1}+e_{3} \prec e_{2}+e_{3} \prec e_{1}+e_{2}+e_{3}
$$

The height, $\hbar(x)$ of an element $x$ in a finite totally ordered set is $x$ 's place in that order. For a set of elements $A$ we denote $\hbar(A)=\sum_{x \in A} \hbar(x)$.

If $T \subseteq \mathbb{Z}_{2}^{n}$, then its initial segment of size $a$, denoted $I S(a, T)$, is the set of the $a$ smallest elements of $T$ in the lexicographic order. We use the abbreviation $I S(a)=I S\left(a, \mathbb{Z}_{2}^{n}\right)$ for $n \in \mathbb{N}$ large enough.

\subsection{The Hopf-Stiefel Function}

For the reader's convenience we prove the following observation of Bollobás and Leader [1].

Proposition 3. For two initial segments $I S(a), I S(b) \subseteq \mathbb{Z}_{2}^{n}$, the sum $I S(a)+I S(b)$ is an initial segment as well.

Proof. For $z \prec x+y$, we claim that $z=x^{\prime}+y^{\prime}$ for some $x^{\prime} \preceq x$ and $y^{\prime} \preceq y$. Let $i \in \mathbb{N}$ be largest index such that $x_{i}=1$ or $y_{i}=1$. Say $x_{i}=1$. If $z_{i}=0$, then clearly $z \prec x$, so we can take $x^{\prime}=z$ and $y^{\prime}=0$. If $z_{i}=1$, then note that $\left(z-e_{i}\right) \prec\left(x-e_{i}\right)+y$. By induction on $i$, obtain $\left(z-e_{i}\right)=x^{\prime \prime}+y^{\prime \prime}$ for $x^{\prime \prime} \preceq\left(x-e_{i}\right)$ and $y^{\prime \prime} \preceq y$, and choose $x^{\prime}=x^{\prime \prime}+e_{i}$ and $y^{\prime}=y^{\prime \prime}$.

The Hopf-Stiefel binary function $a \circ b$ can be defined on $\mathbb{N} \times \mathbb{N}$ as follows:

$$
a \circ b=|I S(a)+I S(b)|
$$

Proposition 3 can be restated as: $I S(a)+I S(b)=I S(a \circ b)$. This definition is relevant for us for the following reason. The cardinality of a sumset of two sets of given cardinalities is minimized by taking the two sets to be initial segments:

$$
a \circ b=\min \left\{|A+B|\left|A, B \in \mathbb{Z}_{2}^{n},\right| A|=a,| B \mid=b\right\}
$$


Note that here the sets are not required to be affinely generating. This result can be deduced by the technique of compressions as we discuss below. See Lemma 6

In particular, taking $A=I S(a)$ and $B=I S\left(b_{1}\right) \cup\left(e_{n}+I S\left(b_{2}\right)\right)$ for $n$ large enough, one can verify the sub-distributive law:

$$
a \circ\left(b_{1}+b_{2}\right) \leq a \circ b_{1}+a \circ b_{2}
$$

Similarly, one can deduce the recursive relations for $a, b \leq 2^{n}$ :

$$
\begin{array}{r}
a \circ\left(2^{n}+b\right)=2^{n}+a \circ b \\
\left(2^{n}+a\right) \circ\left(2^{n}+b\right)=2^{n+1}
\end{array}
$$

These two formulas can be taken as an alternative definition of the Hopf-Stiefel function [27].

The function first arose in works of Hopf [20] and Stiefel [35. They used tools from algebraic topology to prove that $a \circ b$ provides a lower bound for solutions of the Hurwitz problem, concerning real quadratic forms (see 34]). The relation to set addition in $\mathbb{Z}_{2}^{n}$ was given by Yuzvinsky [39. As it turns out, the Hopf-Stiefel function arises in the study of several more problems in various contexts. There is also a base- $p$ analog of the this function for $p>2$, see [7. For a survey, see 9 .

\subsection{Compressions}

For $I=\left\{i_{1}, i_{2}, \ldots\right\} \subseteq[n]$, denote $H_{I}=\left\langle 0, e_{i_{1}}, e_{i_{2}}, \ldots\right\rangle \triangleleft \mathbb{Z}_{2}^{n}$. As usual, if $H$ is a subgroup of $G$, we denote by $G / H$ the collection of all $H$-cosets in $G$. The $I$-compression of a subset $A \subseteq \mathbb{Z}_{2}^{n}$ is defined by:

$$
C_{I}(A)=\bigcup_{T \in \mathbb{Z}_{2}^{n} / H_{I}} I S(|A \cap T|, T)
$$

In words, in every $H_{I}$-coset $T$ we replace the elements of $A \cap T$ by a same-cardinality initial segment, with respect to the lexicographic order. We say $A$ is compressed with respect to $I$, or $I$-compressed, if $C_{I}(A)=A$. In particular, lexicographic initial segments of $\mathbb{Z}_{2}^{n}$ are exactly all $[n]$-compressed sets.

Example 4. $C_{\{1,2,3\}}\left(\left\{0, e_{1}, e_{2}, e_{3}, e_{4}\right\}\right)=\left\{0, e_{1}, e_{2}, e_{1}+e_{2}, e_{4}\right\}$.

This notion of compression is closely related to the operation bearing the same name from extremal set theory (see, e.g., [12]). A subset of $\mathbb{Z}_{2}^{n}$ naturally corresponds to a family, a.k.a. setsystem, $\mathcal{F}$ of subsets of $[n]$. We freely move between these terminologies if no confusion can occur. An $\{i\}$-compression corresponds to the push-down operator $T_{i}$, which replaces $J \in \mathcal{F}$ by $J \backslash\{i\}$ provided that $J \backslash\{i\} \notin \mathcal{F}$. If $\mathcal{F}$ is $\{i\}$-compressed for each $i$, then it is closed under taking subsets and is called a downset. The shift operator $S_{i j}$ replaces $j$ by $i$ wherever possible. Namely, for every $J$ with $i, j \notin J$ it replaces $J \cup\{j\}$ by $J \cup\{i\}$ given that the former belongs to $\mathcal{F}$ and the latter doesn't. We say that $\mathcal{F}$ is shift-minimal if it is invariant to all shifts $S_{i j}$ where $i<j$. One can check that being $\{i, j\}$-compressed for all $i, j \in[n]$ corresponds to being a shift-minimal downset.

Compression can simplify matters substantially, while preserving several useful features of the set-system. Here are some observations about compressions. These and others are found in [17. The proofs are straightforward, working coset by coset.

Lemma 5 (Properties of compressions). Suppose $A \subseteq \mathbb{Z}_{2}^{n}$ and $I \subseteq[n]$.

(1) $\left|C_{I}(A)\right|=|A|$.

(2) $C_{I}(A)$ is $I$-compressed.

(3) $\hbar\left(C_{I}(A)\right) \leq \hbar(A)$ with equality iff $A$ is I-compressed.

(4) An I-compressed set is $J$-compressed for all $J \subseteq I$.

(5) $C_{I}(A) \subseteq C_{I}(B)$ for all $A \subseteq B$.

Compressions behave well on sumsets. By Proposition 3, one can deduce that the sum of two $I$-compressed subsets is $I$-compressed too. The following well-known lemma deals with the compression of a sum of two general subsets. For the sake of completeness, we prove it here, following [1] and [17. 
Lemma 6 (Sumset compression). Suppose $A, B \subseteq \mathbb{Z}_{2}^{n}$ and $I \subseteq[n]$. Then

$$
C_{I}(A)+C_{I}(B) \subseteq C_{I}(A+B) .
$$

Consequently $\left|C_{I}(A)+C_{I}(B)\right| \leq|A+B|$.

Proof. We use a double induction, on $|I|$ and on $\hbar(A)+\hbar(B)$. For the induction step, suppose that for some $J \subsetneq I$ either $A$ or $B$ is not $J$-compressed. In this case

$C_{I}(A)+C_{I}(B)=C_{I}\left(C_{J}(A)\right)+C_{I}\left(C_{J}(B)\right) \subseteq C_{I}\left(C_{J}(A)+C_{J}(B)\right) \subseteq C_{I}\left(C_{J}(A+B)\right)=C_{I}(A+B)$

Both inclusions are by the induction hypothesis: the first one since $\hbar\left(C_{J}(A)\right)+\hbar\left(C_{J}(B)\right)<\hbar(A)+$ $\hbar(B)$ by property (3) of Lemma 5, and the second one since $|J|<|I|$ and by property (5). The equalities are by property (4).

It only remains to verify the lemma for $A$ and $B$ that are both $J$-compressed for all $J \subsetneq I$. We start with the simpler case $n=|I|$.

What are the subsets of $G=\mathbb{Z}_{2}^{n}$ that are $J$-compressed for all $J \subsetneq[n]$ ? By property (4), all initial segments are such. If $S \subseteq G$ is not an initial segment, then necessarily $x \notin S$ and $y \in S$ for some consecutive $x \prec y$. The only consecutive pair in $G$ that is not contained in a proper $H_{J}$-coset is $\left(e_{1}+\ldots+e_{n-1}\right) \prec e_{n}$. One can verify, for example by $S$ being $[2 \ldots n]$-compressed, that the only such set is $S=H_{[n-1]} \backslash\left\{e_{1}+\ldots+e_{n-1}\right\} \cup\left\{e_{n}\right\}$. In conclusion, it is enough to check the case where $A$ and $B$ are initial segments or equal to $S$. Now there are four cases to consider:

1. If both $A$ and $B$ are initial segments, then by Proposition $3 A+B$ is an initial segment too. $\Rightarrow C_{I}(A)+C_{I}(B)=A+B=C_{I}(A+B)$

2. If $A=B=S$, then note that $|S| \leq|S+S|$ and $C_{I}(S)=H_{[n-1]}$. $\Rightarrow C_{I}(S)+C_{I}(S)=C_{I}(S) \subseteq C_{I}(S+S)$

3. If $B=S$ and $A$ is an initial segment with $|A| \leq|S|$, then $A=C_{I}(A) \subseteq C_{I}(S)=H_{[n-1]}$. $\Rightarrow C_{I}(A)+C_{I}(S)=C_{I}(S) \subseteq C_{I}(A+S)$

4. If $B=S$ and $A$ is an initial segment with $|A|>|S|$ then $|A|+|S|>|G|$.

This means $A+S=G$, as the reader may verify by a standard pigeonhole argument. $\Rightarrow C_{I}(A)+C_{I}(S)=G=C_{I}(G)=C_{I}(A+S)$

The case $n>|I|$ is implied by the case $n=|I|$ :

$$
\begin{aligned}
C_{I}(A)+C_{I}(B) & =\bigcup_{H_{c} \in G / H_{I}}\left(\left(C_{I}(A)+C_{I}(B)\right) \cap H_{c}\right) \\
& =\bigcup_{H_{c} \in G / H_{I}} \bigcup_{H_{a}+H_{b}=H_{c}}\left(\left(C_{I}(A) \cap H_{a}\right)+\left(C_{I}(B) \cap H_{b}\right)\right) \\
& =\bigcup_{H_{c} \in G / H_{I}} \bigcup_{H_{a}+H_{b}=H_{c}}\left(C_{I}\left(A \cap H_{a}\right)+C_{I}\left(B \cap H_{b}\right)\right) \\
& \subseteq \bigcup_{H_{c} \in G / H_{I}} \bigcup_{H_{a}+H_{b}=H_{c}} C_{I}\left(\left(A \cap H_{a}\right)+\left(B \cap H_{b}\right)\right) \\
& \subseteq \bigcup_{H_{c} \in G / H_{I}} C_{I}\left(\bigcup_{H_{a}+H_{b}=H_{c}}\left(\left(A \cap H_{a}\right)+\left(B \cap H_{b}\right)\right)\right) \\
& =\bigcup_{H_{c} \in G / H_{I}} C_{I}\left((A+B) \cap H_{c}\right) \\
& =\bigcup_{H_{c} \in G / H_{I}}\left(C_{I}(A+B) \cap H_{c}\right) \\
& =C_{I}(A+B) .
\end{aligned}
$$

The first and second inequalities are simply dividing into cases, according to the involved $H_{I^{-}}$ cosets. The third one holds because compressions work coset-wise. Then there is inclusion by the assumption on the case $I=[n]$, applied to our $H_{I}$ and translated to the relevant $H_{I}$-cosets. And then, inclusion of initial segments, because the union is at least as large as each of its components. The three remaining equalities are similar to the first three. 


\subsection{Compressions that Preserve Affine Generation}

As Lemma 6] shows, in the problems we consider here, compressing the sets under consideration can only improve our objective function. However, we are restricting ourselves to affinely generating sets and compression may destroy this property (e.g., Example 4). Therefore, our strategy is to keep compressing as long as affine generation is maintained. To this end we introduce the following definition.

Suppose that $A \supseteq E$, where $E=\left\{0, e_{1}, e_{2}, \ldots, e_{n}\right\}$ is the standard affine basis of $\mathbb{Z}_{2}^{n}$. If $A$ is $I$-compressed for every $I$ such that $C_{I}(A) \supseteq E$, we say that $A$ is $\langle\langle E\rangle\rangle$-compressed. Note that by part (3) of Lemma 5 every set $A$ containing $E$, can be turned into an $\langle\langle E\rangle\rangle$-compressed set by a finite sequence of such compressions. It turns out that $\langle\langle E\rangle\rangle$-compressed sets are very structured.

Lemma 7 (Structure of $\langle\langle E\rangle\rangle$-compressed sets). Let $A \subseteq \mathbb{Z}_{2}^{n}$ be an $\langle\langle E\rangle\rangle$-compressed set.

(1) A is a shift-minimal downset.

(2) A contains a subgroup of maximal size $H \triangleleft \mathbb{Z}_{2}^{n}$ of the form $H=\left\langle 0, e_{1}, \ldots, e_{h}\right\rangle$.

(3) $A$ is $\{1, \ldots, h, h+i\}$-compressed for every $1 \leq i \leq m=\operatorname{codim} H$.

(4) $A \subseteq H+E$, i.e. $A=H \cup A_{1} \cup A_{2} \cup \ldots \cup A_{m}$ where $A_{i}=A \cap\left(e_{h+i}+H\right)$.

(5) For $1 \leq i \leq m, 0<\left|A_{i}\right|<|H|$.

(6) For $1 \leq i<j \leq m,\left|A_{i}\right|+\left|A_{j}\right| \leq|H|$.

(7) If $m>1$, then $|A| \leq\left(1+\frac{m}{2}\right)|H|$.

Proof. The proofs are fairly straightforward.

(1) It is a simple observation that both $\{i\}$-compressions and $\{i, j\}$-compressions preserve $E \subseteq A$. Hence $A$ must already be compressed with respect to these sets, i.e., a shift-minimal downset.

(2) Let $h$ be the maximal dimension of a subgroup contained in $A$. As shown below in Lemma 8 , a subgroup of dimension $h$ must contain an element of Hamming weight at least $h$. By shift-minimality $e_{1}+e_{2}+\ldots+e_{h} \in A$, and by the downset property $H=\left\langle 0, e_{1}, \ldots, e_{h}\right\rangle \subseteq A$.

(3) Denote $I=\{1, \ldots, h, h+i\}$. The sets $H \cup\left\{e_{h+i}\right\}$ and $\left\{e_{h+j}\right\}$ for $j \neq i$ are initial segments of

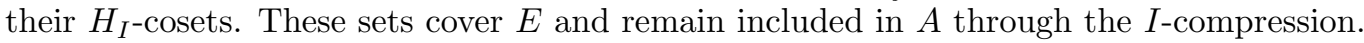

(4) By the downset property, it is sufficient to show $e_{h+i}+e_{h+j} \notin A$ for each $1 \leq i<j \leq m$. Indeed, if $A$ contains $e_{h+i}+e_{h+j}$ then it contains $e_{h+i}+H$ by being $\{1, \ldots, h, h+j\}$-compressed. This implies $H \cup\left(e_{h+i}+H\right) \subseteq A$, contrary to the maximality of the subgroup $H$ in $A$.

(5) For the lower bound note that $e_{h+i} \in E \subseteq A$. On the other hand, if $\left|A_{i}\right|=|H|$ then $H \cup\left(e_{h+i}+H\right) \subseteq A$, contrary, again, to the maximality of $H$.

(6) Note that $e_{h+j} \in A$, while some lexicographically smaller elements in $e_{h+i}+H$ are not contained in $A$. Therefore $A$ can't be $I$-compressed for $I=\{1, \ldots, h, h+i, h+j\}$. Since it is $\langle\langle E\rangle\rangle$-compressed, this means $e_{h+j} \notin C_{I}(A)$. Equivalently, $\left|A \cap H_{I}\right| \leq 2|H|$, which leads to our claim.

(7) If $\left|A_{i}\right| \leq \frac{1}{2}|H|$ for every $i$, clearly $|A|=|H|+\sum_{i=1}^{m}\left|A_{i}\right| \leq\left(1+\frac{m}{2}\right)|H|$. Otherwise, $\left|A_{i}\right|>\frac{1}{2}|H|$ for some $i$, thus $\left|A_{j}\right| \leq|H|-\left|A_{i}\right|<\frac{1}{2}|H|$ for every $j \neq i$. So $\left|A_{i}\right|+\left|A_{j}\right| \leq|H|$ for some $i$ and $j$, and the remaining $A_{j}$ 's are no bigger than $\frac{1}{2}|H|$.

Lemma 8. Let $H$ be an $h$-dimensional subgroup of $\mathbb{Z}_{2}^{n}$. Then $H$ contains an element of Hamming weight at least $h$.

Proof. If $h=n$, take $e_{1}+e_{2}+\ldots+e_{n}$. Otherwise, there exists a basis element $e_{i}$ such that $e_{i} \notin H$. In this case, moving from $H$ to $C_{\{i\}}(H)$ simply deletes $e_{i}$ from the standard basis representations of $H$ 's elements, thereby not increasing their Hamming weights. Now note that $C_{\{i\}}(H)$ is an $h$-dimensional subgroup of $\left\langle 0, e_{1}, \ldots, e_{i-1}, e_{i+1}, \ldots, e_{n}\right\rangle$, and by induction on $n$ contains an element of Hamming weight at least $h$. 


\section{The Freiman-Ruzsa Theorem in $\mathbb{Z}_{2}^{n}$}

For $A \subseteq \mathbb{Z}_{2}^{n}$ we refer to $|\langle A\rangle| /|A|$ as $A$ 's spanning constant and to $K=|A+A| /|A|$ as its doubling constant. The Freiman-Ruzsa theorem gives an upper bound on the spanning constant in terms of $K$. We first review the theorem and some of its quantitative aspects. Then we calculate the bound explicitly, and in particular we determine its correct asymptotics which turns out to be $\Theta\left(2^{2 K} / K\right)$. We present the proof in two stages, starting with the asymptotic estimates. We find this presentation convenient, since the proof of the asymptotic bound already contains our main ideas.

\subsection{Brief Review of the Freiman-Ruzsa Theorem}

Freiman's celebrated theorem [14] states that if $A \subset \mathbb{Z}$ is a finite subset with $|A+A| \leq K|A|$, then $A$ is included in a generalized arithmetic progression, whose size (relative to $|A|$ ) and dimension are bounded. The bounds depend only on $K$ and not on $|A|$. Ruzsa [28, 29, has made crucial contributions to this area. More recently much work was done on similar problems where $\mathbb{Z}$ is replaced by other groups. In particular Ruzsa 31] proved the analogous result for abelian torsion groups. See [37] for a nice exposition.

Theorem 9 (Ruzsa). Let $G$ be an abelian group in which every element has order at most $r$. If $A$ is a finite subset of $G$ with $|A+A| \leq K|A|$, then $A$ is contained in a coset of a subgroup $H \triangleleft G$ of size $|H| \leq f(r, K)|A|$, where

$$
f(r, K) \leq K^{2} r^{K^{4}} .
$$

Better estimates on $f(r, K)$ were subsequently found. We denote by $F(r, K)$ the smallest bound for which this statement holds. Note that $F(r, K)$ is non-decreasing in $K$ and $F(r, 1)=1$.

By considering the case where $A$ is an affine basis of $\mathbb{Z}_{r}^{2(K-1)}$ we see that $F(r, K) \geq r^{2 K-O(\log K)}$ (see Example 11 below). This suggests the following conjecture [31.

Conjecture 10 (Ruzsa). For some $C \geq 2$ we have $F(r, K) \leq r^{C K}$.

In an attempt to understand the role of torsion in these phenomena, much work was dedicated to the special case $r=2$, where $G=\mathbb{Z}_{2}^{n}$. This work is also motivated by the role that $\mathbb{Z}_{2}^{n}$ plays in discrete mathematics and in particular in coding theory [3. We introduce the following notation:

$$
F(K)=F(2, K)=\sup \left\{\frac{|\langle A\rangle|}{|A|} \mid A \subseteq \mathbb{Z}_{2}^{n}, n \in \mathbb{N}, \frac{|A+A|}{|A|} \leq K\right\}
$$

As already observed by Ruzsa [5], for $r=2$ his method gives somewhat more, namely $F(K) \leq$ $K 2^{\lfloor K\rfloor^{3}-1}$. Later work by Green and Ruzsa [16] gave $F(r, K) \leq K^{2} r^{2 K^{2}-2}$, which was again refined for $r=2$ to $F(K) \leq 2^{O\left(K^{3 / 2} \log K\right)}$ by Sanders 32. Using compressions, Green and Tao 17] were able to prove $F(K) \leq 2^{2 K+O(\sqrt{K} \log K)}$. Note that this confirms Conjecture 10 for $r=2$. The best bound so far is due to Konyagin [23] who further improved this method to derive $F(K) \leq 2^{2 K+O(\log K)}$.

The range of small $K$ has received some attention as well. In the sub-critical range $K<2$, the exact value of $F(K)$ is known to be $F(K)=K$ for $1 \leq K<7 / 4$ and $F(K)=\frac{8}{7} K$ for $7 / 4 \leq K<2$. See [6, 17, 19, 26, 41]. For $K \leq 12 / 5$ we have $F(K) \leq(2 K-1) /\left(3 K-K^{2}-1\right)$ and for $12 / 5<K<4$, a recursive formula is available. See [5].

The following simple construction [31] provides a lower bound on $F(K)$.

Example 11 (Independent Points). Consider the subset:

$$
A_{[t]}=\left\{0, e_{1}, e_{2}, \ldots, e_{t}\right\} \subseteq \mathbb{Z}_{2}^{t}
$$

Here, for $t \in \mathbb{N}$ we have $F\left(\frac{\left(\begin{array}{c}t \\ 2\end{array}\right)+t+1}{t+1}\right) \geq \frac{2^{t}}{t+1}$, and by monotonicity one can obtain:

$$
F(K) \geq \frac{1}{4 K} 2^{2 K}(1-o(1))
$$




\subsection{Asymptotics of $F(K)$}

We first prove a new upper bound, which coincides with the construction in Example 11 for $t \in \mathbb{N}$.

Theorem 12. $F\left(\frac{\left(\begin{array}{l}t \\ 2\end{array}\right)+t+1}{t+1}\right) \leq \frac{2^{t}}{t+1}$ holds for $2 \leq t \in \mathbb{R}$. Consequently,

$$
F(K) \leq \frac{1}{2 K} 2^{2 K}(1-o(1))
$$

The exponential term $2^{2 K}$ is as in [17, 23, but the polynomial coefficient $1 / K$ is new. Thus it re-proves Conjecture 10 for $r=2$ with $C=2$. This bound and Example 11 determine the asymptotics of $F(K)$ up to a factor of 2 . In the next section we calculate $F(K)$ exactly, and show that the gap is unavoidable and results from the oscillations in $F(K)$.

Proof. For an affinely generating subset $A \subset G=\mathbb{Z}_{2}^{n}$, it is sufficient to prove:

$$
|A|=\frac{t+1}{2^{t}}|G| \Rightarrow|A+A| \geq \frac{\left(\begin{array}{l}
t \\
2
\end{array}\right)+t+1}{2^{t}}|G|
$$

where $2 \leq t \in \mathbb{R}$. Since both expressions are monotone in $t$, the theorem follows.

As in [17, the main tool is reduction to compressed sets of some sort. First, since $\langle A\rangle=G$ we can assume that $A$ contains an affine basis for $G$. But $|A|,|A+A|$ are not affected by invertible affine transformations, so we may assume without loss of generality $E \subseteq A$, where $E=\left\{0, e_{1}, e_{2}, \ldots, e_{n}\right\}$ is the standard affine basis of $G$. Now we assume without loss of generality that $A$ is $\langle\langle E\rangle\rangle$-compressed. Indeed, supposing (1) holds for $\langle\langle E\rangle\rangle$-compressed subsets, we proceed to general subsets inducting on $\hbar(A)$. Let $I \subseteq[n]$ be a set such that $E \subseteq C_{I}(A) \neq A$. By Lemma $6,\left|C_{I}(A)+C_{I}(A)\right| \leq|A+A|$ while $\left|C_{I}(A)\right|=|A|$, so $A$ satisfies $(1)$ provided that $C_{I}(A)$ does. The inductive argument applies, since $\hbar(A)>\hbar\left(C_{I}(A)\right)$ by Lemma $5(3)$.

We continue the proof using the structure of $\langle\langle E\rangle\rangle$-compressed sets. As in Lemma 7] let $H \subseteq A$ be a maximal subgroup, $h=\operatorname{dim} H, m=\operatorname{codim} H$ and $A_{i}=A \cap\left(e_{h+i}+H\right)$ for $1 \leq i \leq m$. By Lemma $7(7),|A| \leq(1+m / 2)|H|$, and an upper bound on $m$ is given by

$$
\frac{1+\frac{m}{2}}{2^{m}} \geq \frac{|A|}{|G|}
$$

where the case $m=1$ follows from the assumption $2 \leq t$.

Given $m$, Lemma 7(4) gives a decomposition of $A$ into $m+1$ parts, and we use it to show that $A+A$ is at least $\sim m / 2$ times larger than $A$. This is shown by the following calculation, where all indices go from 1 to $m$ and all unions are disjoint:

$$
\begin{aligned}
A & =H \cup \bigcup_{i} A_{i} \\
\Rightarrow \quad A+A & =H \cup \bigcup_{i}\left(A_{i}+H\right) \cup \bigcup_{i<j}\left(A_{i}+A_{j}\right) \\
\sum_{i<j}\left|A_{i}+A_{j}\right| & \geq \sum_{i<j} \max \left(\left|A_{i}\right|,\left|A_{j}\right|\right) \geq \sum_{i<j} \frac{\left|A_{i}\right|+\left|A_{j}\right|}{2}=\frac{m-1}{2} \sum_{i}\left|A_{i}\right|=\frac{m-1}{2}(|A|-|H|) \\
\Rightarrow \quad|A+A| & \geq|H|+m|H|+\frac{m-1}{2}(|A|-|H|)=\frac{m+3}{2} \cdot \frac{|G|}{2^{m}}+\frac{m-1}{2}|A|
\end{aligned}
$$

The right-hand side is decreasing in $m$ in the real interval where $((m+3) \log 2-1) / 2^{m}>|A| /|G|$. This interval includes the range of our interest, which is $(m / 2+1) / 2^{m} \geq|A| /|G|=(t+1) / 2^{t}$, or equivalently $m \leq t-1$. Thus, we obtain a lower bound on $|A+A|$ by evaluating this expression at $t-1$, namely:

$$
|A+A| \geq \frac{(t-1)+3}{2^{(t-1)+1}}|G|+\frac{(t-1)-1}{2} \cdot \frac{t+1}{2^{t}}|G|=\frac{\left(\begin{array}{l}
t \\
2
\end{array}\right)+t+1}{2^{t}}|G|
$$




\subsection{Exact Calculation of $F(K)$}

Theorem 2, which we will shortly prove, provides an explicit formula of $F(K)$. This enables one to rederive the asymptotics of $F(K)$, and to deduce the following corollary.

Corollary 13. Both bounds in the asymptotic inequalities

$$
\frac{1}{4 K} 2^{2 K}(1-o(1)) \leq F(K) \leq \frac{1}{2 K} 2^{2 K}(1-o(1))
$$

are sharp up to the o(1) terms.

It also settles the following conjecture of Diao [6].

Corollary 14. $F(K)$ is a piecewise linear function.

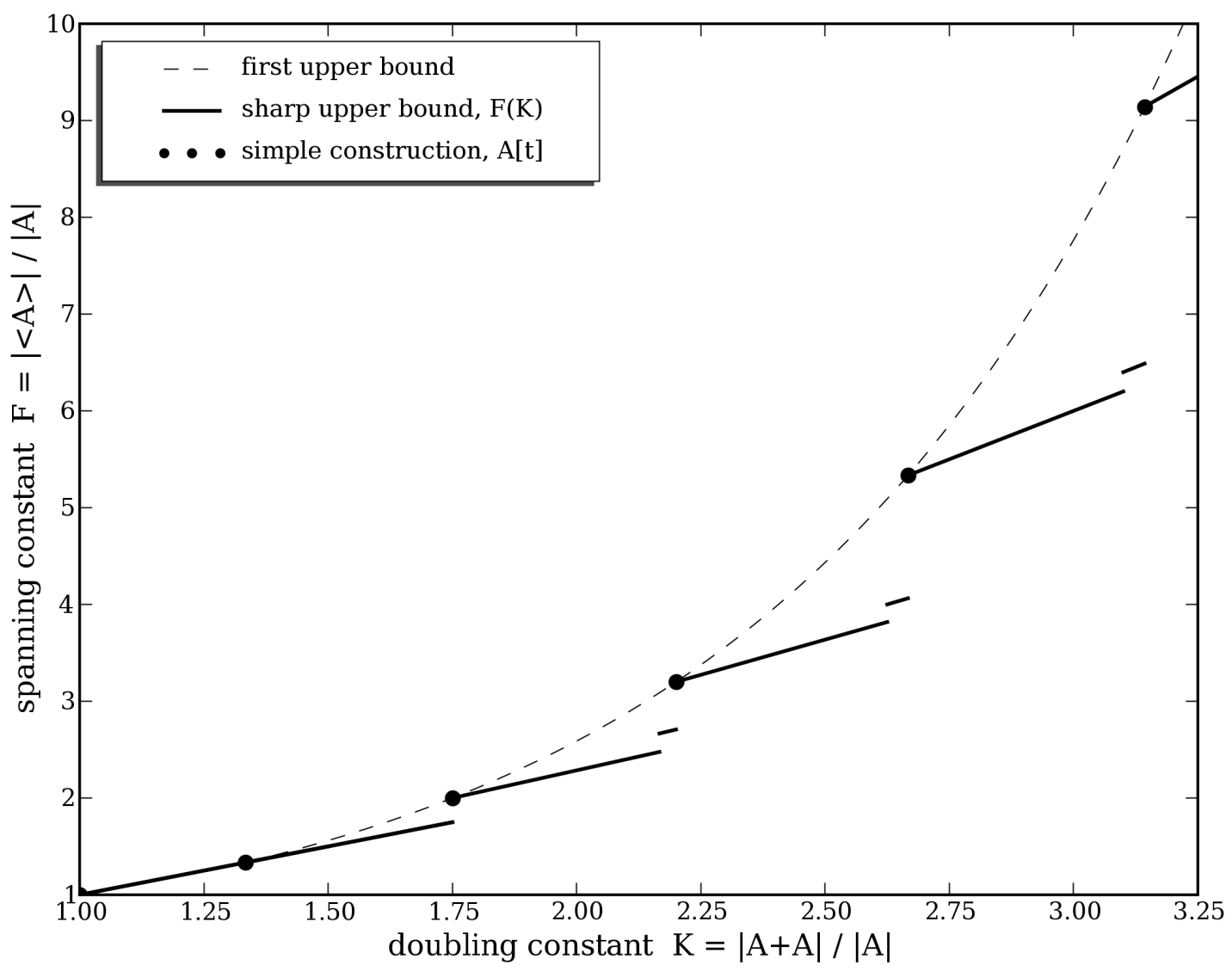

Figure 1: An illustration of $F(K)$

In order to calculate $F(K)$, it is useful to consider a related function $\tilde{K}(\tilde{F})$, which is defined for rational numbers of the form $\tilde{F}=2^{a} / b \geq 1$.

$$
\tilde{K}(\tilde{F})=\inf \left\{\frac{|A+A|}{|A|} \mid A \subseteq \mathbb{Z}_{2}^{n}, n \in \mathbb{N}, \frac{|\langle A\rangle|}{|A|}=\tilde{F}\right\}
$$

That is, the minimal doubling constant of an affinely generating set of relative size exactly $1 / \tilde{F}$. By definition $F(K)=\sup \{\tilde{F} \mid \tilde{K}(\tilde{F}) \leq K\}$. Theorem 12 asserts $\tilde{K}\left(2^{t} /(t+1)\right) \geq\left(\left(\begin{array}{l}t \\ 2\end{array}\right)+t+1\right) /(t+1)$ 
for real $t \geq 2$, and by Example 11 it is an equality for $t \in \mathbb{N}$. In order to analyze $\tilde{K}(\tilde{F})$, we refine the arguments in the proof of Theorem 12, and elaborate on the construction in Example 11. This yields a better view of the structure of sets with a small doubling constant. We begin by describing the extended example.

Example 15. For non-negative integers $s, t$ such that $s<t$, consider the subset:

$$
A_{[t, s]}=\left\{0, e_{0}, e_{1}, e_{2}, \ldots, e_{t}, e_{0}+e_{1}, e_{0}+e_{2}, \ldots, e_{0}+e_{t-s}\right\} \subseteq \mathbb{Z}_{2}^{t+1}
$$

It is not hard to verify that

$$
\left|A_{[t, s]}\right|=2(t+1)-s, \quad\left|A_{[t, s]}+A_{[t, s]}\right|=2\left(\left(\begin{array}{l}
t \\
2
\end{array}\right)+t+1\right)-\left(\begin{array}{l}
s \\
2
\end{array}\right), \quad\left|\left\langle A_{[t, s]}\right\rangle\right|=2^{t+1} .
$$

Therefore:

$$
\tilde{K}\left(\frac{2^{t}}{t+1-s / 2}\right) \leq \frac{\left(\begin{array}{l}
t \\
2
\end{array}\right)+t+1-\left(\begin{array}{l}
s \\
2
\end{array}\right) / 2}{t+1-s / 2}
$$

This example provides an upper bound on $\tilde{K}(\tilde{F})$ for a discrete sequence of values. When $s=0$ it reduces to Example 11 . However, $\tilde{K}(\tilde{F})$ is not necessarily monotone, so we cannot imitate the conclusion of Example 11 and extend the upper bound to general $\tilde{F}$. Still, the following argument does the work.

Lemma 16 (Sublinearity of $\tilde{K}(\tilde{F}))$. If $F_{1}<F_{2}$ are in $\tilde{K}$ 's domain, then $\frac{\tilde{K}\left(F_{1}\right)}{F_{1}} \geq \frac{\tilde{K}\left(F_{2}\right)}{F_{2}}$.

Proof. Let $F_{2}=2^{a} / b$ for some $a, b \in \mathbb{N}$. Suppose $A_{1} \subseteq \mathbb{Z}_{2}^{n}$ is an affinely generating set of size $\left|A_{1}\right|=2^{n} / F_{1}$. Let $m \in \mathbb{N}$ be large enough such that $a \leq n+m<b 2^{n+m-a}$. Consider $A_{1}^{\prime}=$ $A_{1} \times \mathbb{Z}_{2}^{m}$, and note that $A_{1}^{\prime}$ affinely generates $\mathbb{Z}_{2}^{n+m}$ and $\left|A_{1}^{\prime}\right|=2^{n+m} / F_{1}$. Since $F_{1}<F_{2}$ one can take a subset $A_{2} \subseteq A_{1}^{\prime}$ of cardinality $\left|A_{2}\right|=b 2^{n+m-a}=2^{n+m} / F_{2}$. Moreover, by $m$ 's choice $n+m+1 \leq\left|A_{2}\right|$, so a subset $A_{2}$ which affinely generates $\mathbb{Z}_{2}^{n+m}$ can be chosen. Now from $A_{2}+A_{2} \subseteq \overline{A_{1}^{\prime}}+A_{1}^{\prime}=\left(A_{1}+A_{1}\right) \times \mathbb{Z}_{2}^{m}$,

$$
\frac{\left|A_{1}+A_{1}\right|}{\left|A_{1}\right|} \cdot \frac{1}{F_{1}}=\frac{\left|A_{1}+A_{1}\right|}{2^{n}}=\frac{\left|A_{1}^{\prime}+A_{1}^{\prime}\right|}{2^{n+m}} \geq \frac{\left|A_{2}+A_{2}\right|}{2^{n+m}}=\frac{\left|A_{2}+A_{2}\right|}{\left|A_{2}\right|} \cdot \frac{1}{F_{2}} \geq \frac{\tilde{K}\left(F_{2}\right)}{F_{2}} .
$$

The task is accomplished by taking the infimum over $A_{1}$.

Corollary 17 (Superlinearity of $F(K))$. $\frac{F\left(K_{1}\right)}{K_{1}} \leq \frac{F\left(K_{2}\right)}{K_{2}}$ for every $1 \leq K_{1}<K_{2}$.

Example 15 and Lemma 16 supply an upper bound on $\tilde{K}(\tilde{F})$. The following lemma essentially claims that this bound is sharp.

Lemma 18 (Formula for $\tilde{K}(\tilde{F})$ ). Let $\tilde{F} \geq 1$ be of the form $2^{a} / b$ where $a, b \in \mathbb{N}$, and let $s<t$ be the unique pair of non-negative integers for which

$$
\frac{2^{t}}{t+1-s / 2} \leq \tilde{F}<\frac{2^{t}}{t+1-(s+1) / 2}
$$

Then

$$
\tilde{K}(\tilde{F})=\frac{\left(\begin{array}{l}
t \\
2
\end{array}\right)+t+1-\frac{1}{2}\left(\begin{array}{l}
s \\
2
\end{array}\right)}{2^{t}} \cdot \tilde{F}
$$

Since the function $F(K)$ is basically the inverse of $\tilde{K}(\tilde{F})$, Theorem 2 is a direct consequence of Lemma 18. Indeed, Figure 1 is obtained by transposing the graph in Figure 2, and taking the maximum wherever the result is multivalued. We omit further details.

One can notice that $\tilde{K}(\tilde{F})$ has a more complex structure than $F(K)$. Since Theorem 2 employs the information in Lemma 18 only partially, there may be a quicker way of calculating $F(K)$. Nevertheless, we feel that the detailed description of $\tilde{K}(\tilde{F})$ is interesting in its own right, and may shed light on the non-trivial form of $F(K)$.

The proof of Lemma 18 pursues the analysis in Theorem 12 s proof, involving more reduction steps which preserve $|A|$ without increasing $|A+A|$. Through these reductions the structure of $A$ 


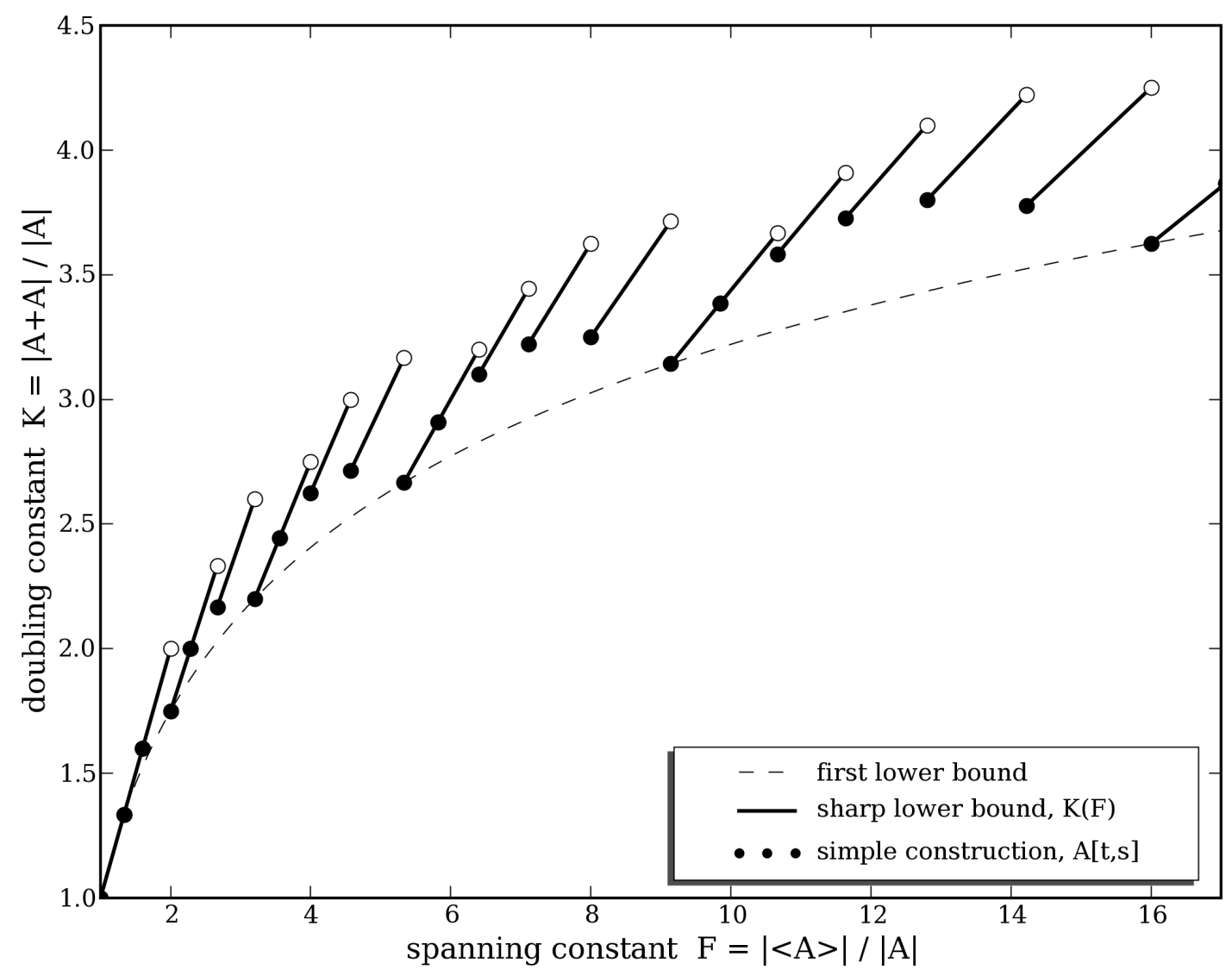

Figure 2: An illustration of $\tilde{K}(\tilde{F})$

becomes similar to Example 15, so that its doubling constant can be calculated explicitly. We start with two reductions which can be formulated separately in terms of integer partitions. All of the following will be motivated and applied later, in the proof of the lemma.

A non-increasing sequence of positive integers $a_{1} \ldots a_{m}$ is an integer partition of $a=\sum_{i} a_{i}$ into $m$ parts, and for short an m-partition of $a$. Recall the Hopf-Stiefel function $a \circ b$ from Section 2. We are interested in the minimum of $\sum_{1 \leq i<j \leq m} a_{i} \circ a_{j}$ over all $m$-partitions of $a$.

A partition $a_{1} \ldots a_{m}$ of $a$ is called compressed if $a_{i}+a_{j}>2^{k} \Rightarrow a_{i} \geq 2^{k}$ for each $k$ and $i<j$. It will be implicit in the proof of Lemma 18, that at least one of the partitions that minimize $\sum_{i<j} a_{i} \circ a_{j}$ is compressed. Here we shall restrict the discussion to compressed partitions.

A partition is called quasi-dyadic if $a_{1} \ldots a_{m-1}$ are powers of 2 . No requirement is made on $a_{m}$. Note that a quasi-dyadic partition is always compressed. Our first reduction basically asserts that the minimum of $\sum_{i<j} a_{i} \circ a_{j}$ is attained by a quasi-dyadic partition.

Lemma 19. A compressed m-partition of a that minimizes $\sum_{i<j} a_{i} \circ a_{j}$ is quasi-dyadic.

Proof. Otherwise, consider the smallest $1 \leq i<m$ for which $a_{i}$ is not a power of 2 , say $2^{k}<$ $a_{i}<2^{k+1}$. Since the partition is compressed, $a_{i}+a_{i+1} \leq 2^{k+1}$. We 'transfer mass' from $a_{i}$ to $a_{i+1}$. Replace $a_{i}$ by $a_{i}^{\prime}=2^{k}$, and replace $a_{i+1}$ by $a_{i+1}^{\prime}=a_{i+1}+a_{i}-2^{k}$. Note that $a_{i}^{\prime} \geq a_{i+1}^{\prime}$ and monotonicity is preserved.

How does this move affect $\sum_{i<j} a_{i} \circ a_{j}$ ? By the choice of $i$, for $j<i$ we have $a_{j}=2^{l}$, where 
$l>k$ as a partition is non-increasing. Thus the terms involving $a_{j}$ are unchanged:

$$
a_{j} \circ a_{i}+a_{j} \circ a_{i+1}=2^{l}+2^{l}=a_{j} \circ a_{i}^{\prime}+a_{j} \circ a_{i+1}^{\prime} .
$$

For $j>i+1$ we know $a_{j} \leq a_{i+1}<2^{k}$. By the recursive definition of the Hopf-Stiefel function, and the sub-distributive law:

$$
\begin{aligned}
a_{j} \circ a_{i}+a_{j} \circ a_{i+1} & =2^{k}+a_{j} \circ\left(a_{i}-2^{k}\right)+a_{j} \circ a_{i+1} \\
& \geq 2^{k}+a_{j} \circ\left(a_{i}-2^{k}+a_{i+1}\right)=a_{j} \circ a_{i}^{\prime}+a_{j} \circ a_{i+1}^{\prime} .
\end{aligned}
$$

Finally, again by the recursive definition the mixed term becomes strictly smaller:

$$
a_{i} \circ a_{i+1}=2^{k}+\left(a_{i}-2^{k}\right) \circ a_{i+1}>2^{k}=a_{i}^{\prime} \circ a_{i+1}^{\prime}
$$

The combination of the last three calculations yields that the sum $\sum_{i<j} a_{i} \circ a_{j}$ can be made smaller by changing the partition, in contradiction to the minimality assumption.

Since $2^{k} \circ a=2^{k}$ for $a \leq 2^{k}$, in the quasi-dyadic case the summation can be simplified :

$$
\sum_{1 \leq i<j \leq m} a_{i} \circ a_{j}=\sum_{1 \leq i<j \leq m} \max \left(a_{i}, a_{j}\right)=\sum_{1 \leq i<j \leq m} a_{i}=\sum_{i=1}^{m}(m-i) \cdot a_{i}
$$

It is natural to conjecture that the minimum is obtained when $a_{1} \ldots a_{m}$ are 'almost' equal. A quasi-dyadic $m$-partition of $a$ is quasi-fair if for some $k \in \mathbb{N}, a_{i} \in\left\{2^{k}, 2^{k-1}\right\}$ for each $1 \leq i \leq m-1$. For example $4+4+2+2+2+1$ and $4+4+4+3$ and $8+4+3$ are some quasi-fair partitions of 15. The following properties of quasi-fair partitions are easily verified:

1. In the above definition one can choose

$$
k=\left\lceil\log _{2}(a / m)\right\rceil,
$$

and then exactly $a_{1} \ldots a_{j}$ exceed $2^{k-1}$ where

$$
j=\left\lceil a / 2^{k-1}\right\rceil-m .
$$

2. For every two positive integers $m \leq a$, there exists a unique quasi-fair $m$-partition of $a$.

3. If $a_{1} \ldots a_{m}$ and $a_{1}^{\prime} \ldots a_{m}^{\prime}$ are the quasi-fair $m$-partitions of $a \leq a^{\prime}$, then $a_{i} \leq a_{i}^{\prime}$ for all $i$.

4. A sub-partition (in the sense of a sub-sequence) of a quasi-fair partition is quasi-fair.

Now we are ready to state the second reduction.

Lemma 20. The minimum of $\sum_{i<j} a_{i} \circ a_{j}$ over all quasi-dyadic m-partitions of a is obtained only by the quasi-fair one.

Proof. This lemma can be verified by induction on $m$. For a partition that minimizes the sum, it is enough to show $a_{1}=2^{k}$ for $k=\left\lceil\log _{2}(a / m)\right\rceil$. By the induction hypothesis $a_{2} \ldots a_{m}$ are quasi-fair, and thus constitute the unique quasi-fair sub-partition we are looking for. By the monotonicity property applied on $a_{2} \ldots a_{m}$, for a competing sequence $a_{1}^{\prime} \ldots a_{m}^{\prime}$ with $a_{1}^{\prime}>a_{1}$, necessarily $a_{i}^{\prime} \leq a_{i}$ for $i \geq 2$, and consequently:

$$
\begin{aligned}
\sum_{i=1}^{m}(m-i) \cdot a_{i} & =\sum_{i=1}^{m}(m-i) \cdot a_{i}+(m-1)\left(\sum_{i=1}^{m} a_{i}^{\prime}-\sum_{i=1}^{m} a_{i}\right) \\
& =\sum_{i=1}^{m}(m-i) \cdot a_{i}^{\prime}-\sum_{i=2}^{m}(i-1)\left(a_{i}-a_{i}^{\prime}\right)<\sum_{i=1}^{m}(m-i) \cdot a_{i}^{\prime}
\end{aligned}
$$

With these reductions in hand, we can complete the calculation of $\tilde{K}(\tilde{F})$. 
Proof. (of Lemma 18 Let $\langle A\rangle=G=\mathbb{Z}_{2}^{n}$. Lemma 18 is proved by showing the following lower bound on $|A+A|$, which is reached by Example 15 and Lemma 16

$$
\frac{t+1-(s+1) / 2}{2^{t}}<\frac{|A|}{|G|} \leq \frac{t+1-s / 2}{2^{t}} \Rightarrow \frac{|A+A|}{|G|} \geq \frac{\left(\begin{array}{l}
t \\
2
\end{array}\right)+t+1-\frac{1}{2}\left(\begin{array}{l}
s \\
2
\end{array}\right)}{2^{t}}
$$

If $|A|>\frac{1}{2}|G|$, then by the pigeonhole principle $A+A=G$, as required in the cases $t=1,2$. Hence we may assume $|A| \leq \frac{1}{2}|G|$ and $t \geq 3$.

We start as in Theorem 12 We first assume without loss of generality that $A$ is $\langle\langle E\rangle\rangle$ compressed, and therefore by Lemma 7 has the following properties:

- There exists a subgroup $H=\left\langle 0, e_{1}, \ldots, e_{h}\right\rangle$ such that $A=H \cup A_{1} \cup A_{2} \cup \ldots \cup A_{m}$, where $A_{i}=A \cap\left(e_{h+i}+H\right)$ and $m=\operatorname{codim} H$.

- $1 / 2^{m}<|A| /|G| \leq\left(1+\frac{m}{2}\right) / 2^{m}$. By the assumptions $\frac{t+2}{2^{t+1}}<|A| /|G| \leq \frac{1}{2}$, we can write $1<m<t$.

- Each $A_{i}$ is a lexicographic initial segment of $e_{i}+H$. Therefore $A$ is uniquely determined by the sequence $a_{1}, \ldots, a_{m}$ where $a_{i}=\left|A_{i}\right|$. Note that $0<a_{i}<2^{h}$.

- By shift-minimality $a_{1} \geq a_{2} \geq \ldots \geq a_{m}$. In other words, $a_{1}, \ldots, a_{m}$ is a partition of $a=$ $|A|-|G| / 2^{m}$

As in Theorem 12, we use these properties to write $A+A$ as a disjoint union of its intersections with $H$-cosets, which are of three forms: $H, H+A_{i}$ and $A_{i}+A_{j}$. Since the $A_{i}$ 's are initial segments of their cosets, the sumsets of the third form can be expressed via the Hopf-Stiefel function:

$$
|A+A|=|H|+m|H|+\sum_{1 \leq i<j \leq m}\left|A_{i}+A_{j}\right|=\frac{m+1}{2^{m}} \cdot|G|+\sum_{1 \leq i<j \leq m} a_{i} \circ a_{j}
$$

This equation makes it interesting to find partitions $a_{1} \ldots a_{m}$ of $a$, that minimize $\sum_{i<j} a_{i} \circ a_{j}$.

We next show that the partition $a_{1} \ldots a_{m}$ is compressed. For $i<j$ and $1 \leq k \leq h$ we exclude the case where $a_{i}<2^{k}<a_{i}+a_{j}$ by the assumption that $A$ is already $\langle\langle E\rangle\rangle$-compressed. For $I=\{1,2, \ldots, k, h+i, h+j\}$, let's examine the set $C_{I}(A) . A_{i}$ is replaced by an initial segment of $e_{h+i}+H$ of size $2^{k}$, and $A_{j}$ is replaced by an initial segment of $e_{h+j}+H$ of size $a_{i}+a_{j}-2^{k}$, which is not empty by assumption. In other words, $a_{i}$ becomes $2^{k}$, and $E \subseteq A$ is preserved.

By Lemmas 19, 20, if $|A+A|$ is minimal then $a_{1} \ldots a_{m}$ is the quasi-fair quasi-dyadic $m$-partition of $a=|A|-|G| / 2^{m}$. In this situation

$$
|A+A|-\frac{m+1}{2^{m}} \cdot|G|=\sum_{i=1}^{j}(m-i) \cdot 2^{k}+\sum_{i=j+1}^{m}(m-i) \cdot 2^{k-1}=\left[\left(\begin{array}{c}
m \\
2
\end{array}\right)-\frac{1}{2}\left(\begin{array}{c}
m-j \\
2
\end{array}\right)\right] 2^{k}
$$

for $0 \leq j \leq m$ and $0<k<(\operatorname{dim} G-m)$ such that

$$
\frac{m+j-1}{2} \cdot 2^{k}<|A|-\frac{|G|}{2^{m}} \leq \frac{m+j}{2} \cdot 2^{k}
$$

Remark. Note that in the cases $j=m-1$ or $j=m$ we can choose $j^{\prime}=0$ and $k^{\prime}=k+1$ as well. We could avoid this freedom of choice by not permitting $j=0$, but since it does not affect the resulting $|A+A|$, we allow both ways.

All that remains now is to show that, as in Theorem 12 , to minimize $|A+A|$ we should make $m$ as large as possible, i.e., $m=t-1$. The proof is by induction on $t-m$ :

- Suppose $m=t-1$. We check that (2) holds.

$$
\frac{2 m-(s+1)}{2} \cdot \frac{|G|}{2^{m+1}}<|A|-\frac{|G|}{2^{m}} \leq \frac{2 m-s}{2} \cdot \frac{|G|}{2^{m+1}}
$$

Denote $k=\operatorname{dim} G-m-1$ and $j=m-s$, and observe that $0 \leq j \leq m$. Then the above expression for the minimal $|A+A|$ becomes

$$
|A+A|=\frac{m+1}{2^{m}} \cdot|G|+\left[\left(\begin{array}{c}
m \\
2
\end{array}\right)-\frac{1}{2}\left(\begin{array}{c}
m-(m-s) \\
2
\end{array}\right)\right] \cdot \frac{|G|}{2^{m+1}}=\frac{\left(\begin{array}{l}
t \\
2
\end{array}\right)+t+1-\frac{1}{2}\left(\begin{array}{l}
s \\
2
\end{array}\right)}{2^{t}} \cdot|G|
$$


- Suppose $m<t-1$. The above discussion yields a compressed set $A$, such that $a_{1} \ldots a_{m}$ is the quasi-fair quasi-dyadic partition of $|A|-|H|$, and $|A+A|$ is minimal given $m$, and equals:

$$
|A+A|=\frac{m+1}{2^{m}}|G|+\sum_{1 \leq i<j \leq m} a_{i} \circ a_{j}
$$

We show that increasing $m$ makes $|A+A|$ smaller. Denote by $A^{\prime}, H^{\prime}$ and $a_{1}^{\prime} \ldots a_{m+1}^{\prime}$ the corresponding set, subgroup and partition for $m^{\prime}=m+1$. Similarly:

$$
\left|A^{\prime}+A^{\prime}\right|=\frac{m+2}{2^{m+1}}|G|+\sum_{1 \leq i<j \leq m+1} a_{i}^{\prime} \circ a_{j}^{\prime}
$$

Now define $a_{0}=\left|H^{\prime}\right|=|H| / 2=|G| / 2^{m+1}$. Since $a_{1} \ldots a_{m}$ is a quasi-dyadic $m$-partition for $m>1$ and $a_{1}<|H|$, necessarily $a_{0} \geq a_{i}$ and $a_{0} \circ a_{i}=a_{0}$ for all $1 \leq i \leq m$. Hence for the quasi-dyadic $(m+1)$-partition $a_{0} \ldots a_{m}$ :

$$
|A+A|=\left(\frac{m+1}{2^{m}}-\frac{m}{2^{m+1}}\right)|G|+m \cdot a_{0}+\sum_{1 \leq i<j \leq m} a_{i} \circ a_{j}=\frac{m+2}{2^{m+1}}|G|+\sum_{0 \leq i<j \leq m} a_{i} \circ a_{j}
$$

But by Lemma 20 the partition $a_{1}^{\prime} \ldots a_{m+1}^{\prime}$ gives the minimal value for this expression. Moreover, since $a_{0}=\left|H^{\prime}\right|>a_{1}^{\prime}$ these partitions differ and $\left|A^{\prime}+A^{\prime}\right|<|A+A|$.

Remark. An examination of the proof reveals two kinds of reduction steps. Either $A$ is compressed without changing $\langle A\rangle$, or we find a set $A^{\prime}$ where $\left|A^{\prime}\right|=|A|$ and $\left|A^{\prime}+A^{\prime}\right|$ is substantially smaller than $|A+A|$. Hence, the proof actually provides a characterization of the extremal case, up to compressions that preserve $\langle A\rangle$ and $|A+A|$.

\section{Addition of two different sets}

What is the smallest possible cardinality of $A+B$ if $A, B \subseteq G=\mathbb{Z}_{2}^{n}$ are two affinely spanning subsets of given cardinalities? In this section we prove Theorem 1 , which gives an essentially complete answer. In addition we establish a new isoperimetric inequality, which is used in the proof. But first, we make some remarks concerning the theorem.

Remarks (on Theorem 1).

1. Tightness: Consider $(|A| /|G|,|B| /|G|,|A+B| /|G|)$ as a point in $[0,1]^{3}$. The Hamming balls construction shows that the bound goes through the points of the form:

$$
\left(\frac{1+t}{2^{t}}, \frac{1+t+\ldots+\left(\begin{array}{c}
t \\
k
\end{array}\right)}{2^{t}}, \frac{1+t+\ldots+\left(\begin{array}{c}
t \\
k+1
\end{array}\right)}{2^{t}}\right) \quad 0 \leq k<t
$$

An inspection of Figure 3 shows that all points properly inside their convex hull are strictly below the bound, and hence cannot be realized by such sets. In other words, further improvements of the bound will be local in nature.

2. The formulation of the theorem apparently breaks the symmetry and doesn't require $\langle B\rangle=G$. Still, there is an asymmetry in the result as well, and the theorem is of interest mostly when $|A| \leq|B|$. See also the remark after the proof.

3. In order to simplify the statement of the theorem, $t$ is defined as the largest positive integer such that $|A| /|G| \leq(t+1) / 2^{t}$. However, the only assumption on $t$ which the proof actually uses is:

$$
\frac{t+2}{2^{t+1}}<\frac{|A|}{|G|}
$$

The theorem can, therefore, be applied as well with $t$ larger than in the given formulation. As Figure 3 shows, the resulting bound would be weaker, but may still be useful in certain contexts. 


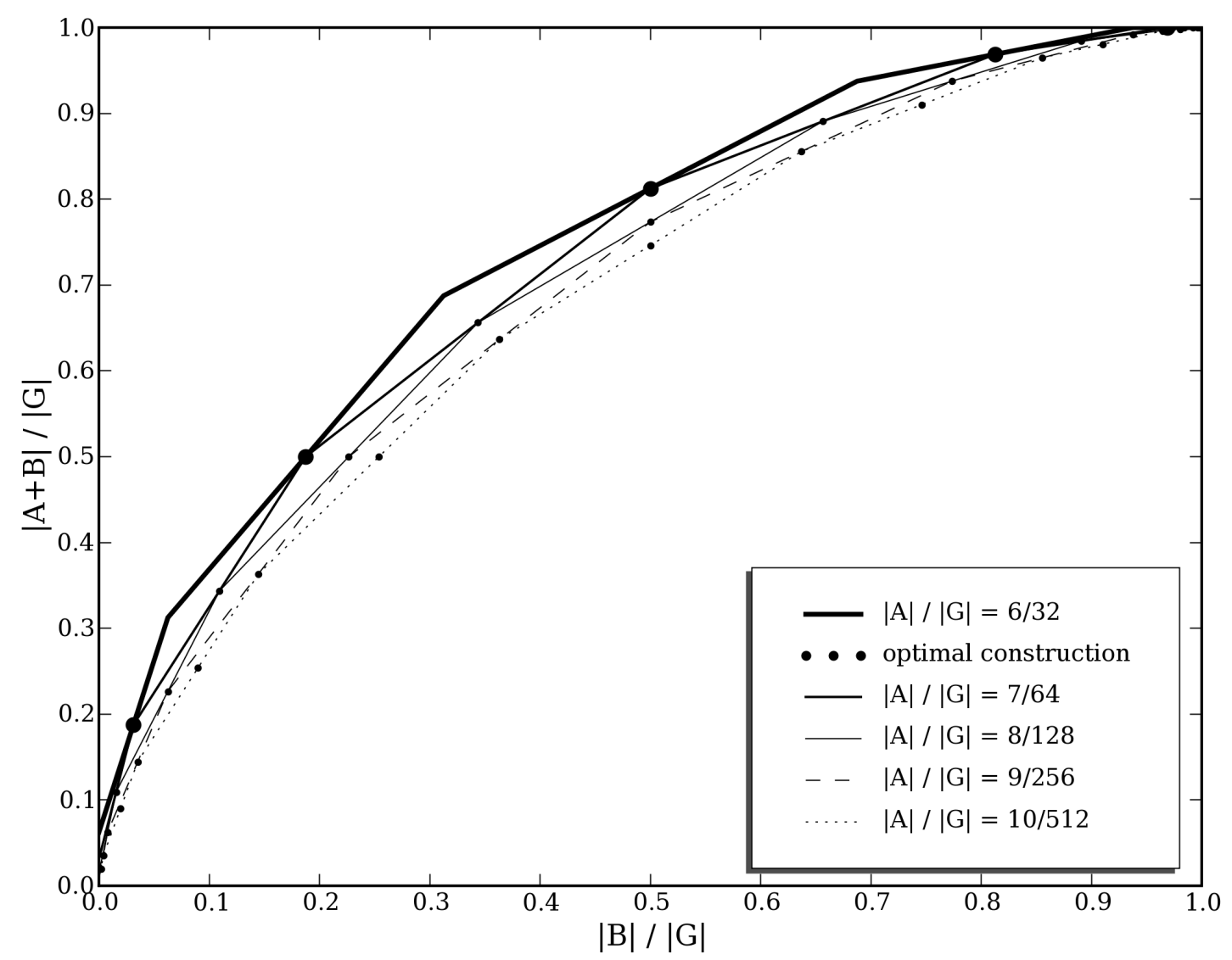

Figure 3: An illustration of the lower bound

Theorem 1 implies that a large enough number of large enough affinely generating sets must add up to the whole group:

Corollary 21. Suppose that $\left\langle A_{1}\right\rangle=\left\langle A_{2}\right\rangle=\ldots=\left\langle A_{m}\right\rangle=G=\mathbb{Z}_{2}^{n}$ with $\left|A_{i}\right| /|G|>(m+2) / 2^{m+1}$ for all $i$. Then $A_{1}+A_{2}+\ldots+A_{m}=G$.

Proof. We repeatedly apply the theorem with $A=A_{i}$ and $B=A_{1}+\ldots+A_{i-1}$ for all $1 \leq i \leq m$ to conclude

$$
\frac{\left|A_{1}+A_{2}+\ldots+A_{i}\right|}{|G|}>\frac{\left(\begin{array}{c}
m+1 \\
0
\end{array}\right)+\left(\begin{array}{c}
m+1 \\
1
\end{array}\right)+\ldots+\left(\begin{array}{c}
m+1 \\
i
\end{array}\right)}{2^{m+1}}
$$

Indeed, in view of remark 3 above and the assumption on the cardinalities, we may choose $t=m+1$, and then $k=i-1$ and $w>0$ by the induction hypothesis. Since $\left|A_{1}+A_{2}+\ldots+A_{m-1}\right|+\left|A_{m}\right|>|G|$ the proof is completed by the pigeonhole principle, $|A|+|B|>|G| \Rightarrow A+B=G$.

The special case of Corollary 21 where all $A_{i}$ are identical is due to Lev [25, following a conjecture of Zemor [40. Taking $\overline{A_{i}}=D_{1}^{m+1} \times \mathbb{Z}_{2}^{n-m+1}$ for each $i$ shows that the assumption on the cardinalities is sharp.

\subsection{An Isoperimetric Inequality}

We are inspired by Frankl's short inductive proof [13] of Harper's theorem [18. 
Theorem 22 (Harper's Inequality). Suppose $A \subseteq \mathbb{Z}_{2}^{n}$.

If for $1 \leq k \leq n$ integer and $0 \leq p \leq 1$ real

$$
|A|=\left(\begin{array}{l}
n \\
n
\end{array}\right)+\left(\begin{array}{c}
n \\
n-1
\end{array}\right)+\ldots+\left(\begin{array}{c}
n \\
k+1
\end{array}\right)+p\left(\begin{array}{l}
n \\
k
\end{array}\right)
$$

then

$$
\left|A+D_{1}^{n}\right| \geq\left(\begin{array}{l}
n \\
n
\end{array}\right)+\left(\begin{array}{c}
n \\
n-1
\end{array}\right)+\ldots+\left(\begin{array}{l}
n \\
k
\end{array}\right)+p\left(\begin{array}{c}
n \\
k-1
\end{array}\right)
$$

In simple terms this theorem says that Hamming balls solve the vertex-isoperimetric problem in the hypercube. However, it also deals, to varying degrees depending on the version of the theorem, with sets of cardinalities strictly between $\left|D_{k-1}^{n}\right|$ and $\left|D_{k}^{n}\right|$. A stronger version would replace the last summand of each expression with $\left(\begin{array}{l}x \\ k\end{array}\right)$ and $\left(\begin{array}{c}x \\ k-1\end{array}\right)$ respectively, where $x \in[k, n]$ is real. The optimal formulation due to Katona [22] and Kruskal [24] is stated in terms of the $k$-cascade representations $\left(\begin{array}{c}a_{k} \\ k\end{array}\right)+\left(\begin{array}{c}a_{k-1} \\ k-1\end{array}\right)+\ldots$ and $\left(\begin{array}{c}a_{k} \\ k-1\end{array}\right)+\left(\begin{array}{c}a_{k-1} \\ k-2\end{array}\right)+\ldots$ respectively. Frankl's method yields all three formulations.

Frankl's proof employs several useful operators on set-systems. As usual, we freely move between the set-theoretic terminology of $2^{[n]}$ and the algebraic language of $\mathbb{Z}_{2}^{n}$. The push-down operator $T_{i}$ and the shift operator $S_{i j}$ have already appeared in Section 2. The upper and the lower shadow operators act on a set-system $\mathcal{F} \subseteq 2^{[n]}$ by

$$
\begin{aligned}
& \delta \mathcal{F}=\{J \cup\{i\} \mid J \in \mathcal{F}, i \notin J\} \\
& \partial \mathcal{F}=\{J \backslash\{i\} \mid J \in \mathcal{F}, i \in J\}
\end{aligned}
$$

respectively. For downsets, the notion of the shadow is close to that of the neighborhood in the theorem. If $C \subseteq \mathbb{Z}_{2}^{n}$ is a non-empty downset, then $C+D_{1}^{n}=\delta C \cup\{0\}$. Note that always $0 \notin \delta A$. Another useful operation on set-systems is classification by $n$, denoted by:

$$
\begin{aligned}
& \mathcal{F}^{-}=\{J \mid J \in \mathcal{F}, n \notin J\} \\
& \mathcal{F}^{+}=\{J \backslash\{n\} \mid J \in \mathcal{F}, n \in J\}
\end{aligned}
$$

When $A \subseteq \mathbb{Z}_{2}^{n}$, we regard $A^{+}$and $A^{-}$as subsets of $\mathbb{Z}_{2}^{n-1}$.

Following Frankl [13, we proceed with two lemmas regarding properties of shifts and shadows.

Lemma 23. Suppose $C \subseteq \mathbb{Z}_{2}^{n}$ is a shift-minimal downset.

(1) $\delta\left(C^{+}\right) \subseteq(\delta C)^{+}=C^{-}$with equality iff $C=\varnothing$

(2) $\delta\left(C^{-}\right)=(\delta C)^{-}$

Proof. Examine the effect of the operators on the representation of some $x \in C$ with the standard basis $e_{1}, \ldots, e_{n}$.

In both $\delta\left(C^{+}\right)$and $(\delta C)^{+}$, some $e_{i}$ is added and $e_{n}$ is removed. However, in $\delta\left(C^{+}\right)$certainly $i \neq n$ since $C^{+}$lives in $\mathbb{Z}_{2}^{n-1}$, while in $(\delta C)^{+}$it is possible that $i=n$. Hence $\delta\left(C^{+}\right) \subseteq(\delta C)^{+}$. By shift-minimality $C$ is closed under these swaps, thus $(\delta C)^{+} \subseteq C^{-}$. Moreover, every element of $C^{-}$is obtained by adding $e_{n}$ and then deleting it, so there is equality. However, $\delta\left(C^{+}\right)$is strictly smaller since $0 \in C^{-} \backslash \delta\left(C^{+}\right)$unless $C$ is empty.

For $\delta\left(C^{-}\right)=(\delta C)^{-}$, note that both sets consist of elements of the form $x+e_{i}$ for $x \in C$ and $i<n$, where $e_{i}$ and $e_{n}$ do not appear in $x$ 's standard representation.

The following lemma is well known. See e.g. 11, 21. Here we prove it as a special case of the compression machinery.

Lemma 24. For all $A \subseteq \mathbb{Z}_{2}^{n}$ and $1 \leq i, j \leq n$ such that $i \neq j$,

(1) $\delta\left(S_{i j} A\right) \subseteq S_{i j}(\delta A)$

(2) $\partial\left(S_{i j} A\right) \subseteq S_{i j}(\partial A)$ 
Proof. By passing from $A$ to $\sum_{i} e_{i}-A$, it is enough to prove only one of the inclusions. Denote $A=\bigcup_{k=0}^{n} A_{k}$ where $A_{k}=A \cap\left(D_{k}^{n} \backslash D_{k-1}^{n}\right)$. Note that we can work with each $A_{k}$ separately. One can write

$$
\delta\left(S_{i j} A_{k}\right)=\left(D_{1}^{n}+C_{i j}\left(A_{k} \cup D_{k-1}^{n}\right)\right) \backslash D_{k}^{n}
$$

and

$$
S_{i j}\left(\delta A_{k}\right)=C_{i j}\left(D_{1}^{n}+\left(A_{k} \cup D_{k-1}^{n}\right)\right) \backslash D_{k}^{n},
$$

yielding our claim by Lemma 6 , since $D_{1}^{n}+C_{i j}(B)=C_{i j}\left(D_{1}^{n}\right)+C_{i j}(B) \subseteq C_{i j}\left(D_{1}^{n}+B\right)$.

Our isoperimetric inequality concerns a family of non-empty downsets $C_{1} \ldots C_{l} \subseteq \mathbb{Z}_{2}^{n}$, rather than a single one. For the volume and the shadow we take the average quantities, denoted by:

$$
\mathrm{E}[C]=\frac{1}{l} \sum_{m=1}^{l}\left|C_{m}\right| \quad \mathrm{E}[\delta C]=\frac{1}{l} \sum_{m=1}^{l}\left|\delta\left(C_{m}\right)\right|
$$

It is hard to make a meaningful statement about these average quantities without limiting the downsets somehow. To see this, consider what happens when each $C_{m}$ is either full or empty. We limit the variability of the downsets by assuming the antichain condition. Namely, we require that for each $i$ and $j, C_{i} \backslash C_{j}$ is an antichain with respect to set-systems inclusion, or equivalently $C_{j} \supseteq \partial C_{i}$.

Proposition 25. Suppose $C_{1} \ldots C_{l} \subseteq \mathbb{Z}_{2}^{n}$ is a family of downsets which satisfies the antichain condition. If

$$
\mathrm{E}[C]=\left(\begin{array}{l}
n \\
0
\end{array}\right)+\left(\begin{array}{l}
n \\
1
\end{array}\right)+\ldots+\left(\begin{array}{c}
n \\
k-1
\end{array}\right)+p\left(\begin{array}{l}
n \\
k
\end{array}\right)
$$

for some integer $k \geq 0$ and real number $0 \leq p<1$, then

$$
\mathrm{E}[\delta C] \geq\left(\begin{array}{l}
n \\
1
\end{array}\right)+\left(\begin{array}{l}
n \\
2
\end{array}\right)+\ldots+\left(\begin{array}{l}
n \\
k
\end{array}\right)+p\left(\begin{array}{c}
n \\
k+1
\end{array}\right)
$$

Since for non-empty downsets $C+D_{1}^{n}=\{0\} \cup \delta C$, the corresponding inequality in the language of neighborhoods is as follows.

Corollary 26. In the setting of Proposition 25, if $C_{1} \ldots C_{l}$ are non-empty then

$$
\mathrm{E}\left[C+D_{1}^{n}\right] \geq\left(\begin{array}{l}
n \\
0
\end{array}\right)+\left(\begin{array}{l}
n \\
1
\end{array}\right)+\ldots+\left(\begin{array}{l}
n \\
k
\end{array}\right)+p\left(\begin{array}{c}
n \\
k+1
\end{array}\right)
$$

Proof. (of Proposition 25 We may assume that the downsets are shift-minimal. Indeed, for each downset $C_{m}$ clearly $S_{i j} C_{m}$ is a downset of the same size, while $\left|\delta\left(S_{i j} C_{m}\right)\right| \leq\left|S_{i j}\left(\delta C_{m}\right)\right|=\left|\delta C_{m}\right|$ by Lemma 24. If $C_{m^{\prime}} \backslash C_{m}$ is an antichain, then $C_{m} \supseteq \partial C_{m^{\prime}}$, hence $S_{i j} C_{m} \supseteq S_{i j}\left(\partial C_{m^{\prime}}\right) \supseteq \partial\left(S_{i j} C_{m^{\prime}}\right)$ by Lemma 24 again, and hence $S_{i j} C_{m^{\prime}} \backslash S_{i j} C_{m}$ is an antichain as well. In conclusion, $S_{i j} C_{1} \ldots S_{i j} C_{l}$ satisfy the antichain condition, $\mathrm{E}[C]=\mathrm{E}\left[S_{i j} C\right]$ and $\mathrm{E}[\delta C] \geq \mathrm{E}\left[\delta\left(S_{i j} C\right)\right]$. After a finite sequence of shifts the downsets are all shift-minimal, since for a proper shift $\sum_{m} \hbar\left(S_{i j} C_{m}\right)<\sum_{m} \hbar\left(C_{m}\right)$.

The case $k=0$ is established separately. Note that in this case $\mathrm{E}[C]<1$, hence $C_{m}=\varnothing$ for some $m$. Actually, this is a sufficient condition for $k=0$, because all other downsets are either $\varnothing$ or $\{0\}$ by the antichain condition. Since $\delta\{0\}=\left\{e_{1}, \ldots, e_{n}\right\}$, clearly $\mathrm{E}[\delta C]=n \cdot \mathrm{E}[C]$ as required.

Following Frankl, we proceed by induction on $n$. By convention $\left(\begin{array}{l}n \\ k\end{array}\right)=0$ for $n<k$. Thus, for $n=0$ the lemma is vacuously satisfied by $\mathrm{E}[\delta C] \geq 0$.

For positive $k$ and $n$, we employ the induction hypothesis on the families $C_{1}^{-} \ldots C_{l}^{-}$and $C_{1}^{+} \ldots C_{l}^{+}$ in $\mathbb{Z}_{2}^{n-1}$. It is easily checked that given a downset $C_{m}$, the sets $C_{m}^{+}$and $C_{m}^{-}$are downsets as well. In addition, if $C_{m^{\prime}} \backslash C_{m}$ is an antichain, then so are its two parts, $C_{m^{\prime}}^{-} \backslash C_{m}^{-}$and $C_{m^{\prime}}^{+} \backslash C_{m}^{+}$, hence the new families satisfy the antichain condition.

By the induction hypothesis on $C_{1}^{+} \ldots C_{l}^{+} \subseteq \mathbb{Z}_{2}^{n-1}$, at least one of the following must hold:

$$
\begin{aligned}
& \mathrm{E}\left[C^{+}\right]<\left(\begin{array}{c}
n-1 \\
0
\end{array}\right)+\left(\begin{array}{c}
n-1 \\
1
\end{array}\right)+\ldots+\left(\begin{array}{l}
n-1 \\
k-2
\end{array}\right)+p\left(\begin{array}{c}
n-1 \\
k-1
\end{array}\right) \\
& \mathrm{E}\left[\delta\left(C^{+}\right)\right] \geq\left(\begin{array}{c}
n-1 \\
1
\end{array}\right)+\left(\begin{array}{c}
n-1 \\
2
\end{array}\right)+\ldots+\left(\begin{array}{c}
n-1 \\
k-1
\end{array}\right)+p\left(\begin{array}{c}
n-1 \\
k
\end{array}\right)
\end{aligned}
$$


Use $\mathrm{E}\left[C^{-}\right]=\mathrm{E}[C]-\mathrm{E}\left[C^{+}\right]$and Pascal's rule in the first case, or $\mathrm{E}\left[C^{-}\right] \geq 1+\mathrm{E}\left[\delta\left(C^{+}\right)\right]$by Lemma 23(1) in the second one, to deduce:

$$
\mathrm{E}\left[C^{-}\right] \geq\left(\begin{array}{c}
n-1 \\
0
\end{array}\right)+\left(\begin{array}{c}
n-1 \\
1
\end{array}\right)+\ldots+\left(\begin{array}{l}
n-1 \\
k-1
\end{array}\right)+p\left(\begin{array}{c}
n-1 \\
k
\end{array}\right)
$$

Note that since $k>0$ each $C_{m}$ is non-empty, so there is proper inclusion in the lemma, which yields the extra 1 in the calculation. By the induction hypothesis on $C_{1}^{-} \ldots C_{l}^{-} \subseteq \mathbb{Z}_{2}^{n-1}$ :

$$
\mathrm{E}\left[\delta\left(C^{-}\right)\right] \geq\left(\begin{array}{c}
n-1 \\
1
\end{array}\right)+\left(\begin{array}{c}
n-1 \\
2
\end{array}\right)+\ldots+\left(\begin{array}{c}
n-1 \\
k
\end{array}\right)+p\left(\begin{array}{c}
n-1 \\
k+1
\end{array}\right)
$$

By Lemma 23, $\mathrm{E}[\delta C]=\mathrm{E}\left[(\delta C)^{-}\right]+\mathrm{E}\left[(\delta C)^{+}\right]=\mathrm{E}\left[\delta\left(C^{-}\right)\right]+\mathrm{E}\left[C^{-}\right]$, hence by Pascal's rule:

$$
\mathrm{E}[\delta C] \geq\left(\begin{array}{l}
n \\
1
\end{array}\right)+\left(\begin{array}{l}
n \\
2
\end{array}\right)+\ldots+\left(\begin{array}{l}
n \\
k
\end{array}\right)+p\left(\begin{array}{c}
n \\
k+1
\end{array}\right)
$$

\subsection{Proof of Lower Bound}

Proof. (of Theorem 1) The general idea is similar to the case $A+A$ discussed in the previous section. By applying various compressions, the sets $A$ and $B$ acquire certain structural properties. These, in turn, allow us to derive estimates on the cardinality of $A+B$.

Lemma 6 asserts that compressions do not increase sumsets: $\left|C_{I}(A)+C_{I}(B)\right| \leq|A+B|$ holds while $\left|C_{I}(A)\right|=|A|$ and $\left|C_{I}(B)\right|=|B|$. Thus, in the search for a lower bound for $|A+B|$, one can first apply a compression $C_{I}$ on $A$ and $B$ simultaneously. Since $\langle A\rangle=G$, we may suppose $E=\left\{0, e_{1}, e_{2}, \ldots, e_{n}\right\} \subseteq A$ and restrict ourselves only to compressions that preserve the inclusion $E \subseteq A$. By Lemma $5(3)$, if a compression $C_{I}$ changes either $A$ or $B$, then $\hbar(A)+\hbar(B)$ strictly decreases. It follows that every sequence of such compressions must terminate. In conclusion, we can assume that both $A$ and $B$ are invariant under these compressions, or for short $\langle\langle E \subseteq A\rangle\rangle$ compressed. This implies that $B$ is $I$-compressed for every $I \subseteq[n]$ such that $A$ is $I$-compressed.

Lemma 7 provides a description of $A$ under this assumption. In particular, $H \subseteq A \subseteq H+E$ for some subgroup $H=\left\langle 0, e_{1}, \ldots, e_{h}\right\rangle$. We next derive some structural properties of $B$.

Lemma 27. Suppose $A, B \subseteq G=\mathbb{Z}_{2}^{n}$ are $\langle\langle E \subseteq A\rangle\rangle$-compressed. Let $H \subseteq A$ be as in Lemma 7 . Consider $G / H \cong \mathbb{Z}_{2}^{m}$ where $m=n-h=\operatorname{codim} H$, with the basis $\left\{e_{h+1}+H, \ldots, e_{h+m}+H\right\}$ and the partial order of the corresponding set-system. For $1 \leq j \leq|H|$ let

$$
C_{j}=\left\{H^{\prime} \in G / H|| B \cap H^{\prime} \mid \geq j\right\}
$$

Then $C_{1} \ldots C_{|H|}$ are downsets, and satisfy the antichain condition.

Proof. By Lemma 7 (3), $A$ is $\{1, \ldots, h, h+i\}$-compressed for $1 \leq i \leq m$, and therefore so is $B$.

Let $H^{\prime} \prec H^{\prime \prime}$ be adjacent $H$-cosets in the partial order. $H^{\prime \prime}=e_{h+i}+H^{\prime}$ for some $1 \leq i \leq m$. Since $B$ is $\{1, \ldots, h, h+i\}$-compressed, $B \cap\left(H^{\prime} \cup H^{\prime \prime}\right)$ must be an initial segment of $H^{\prime} \cup H^{\prime \prime}$. Note that all $H^{\prime}$ elements are lexicographically smaller than those of $H^{\prime \prime}$. Consequently, if $B \cap H^{\prime \prime} \neq \varnothing$ then necessarily $H^{\prime} \subseteq B$. In other words, $H^{\prime \prime} \in C_{1} \Rightarrow H^{\prime} \in C_{|H|}$ for each such pair.

In particular, $C_{j}$ is a downset because $H^{\prime \prime} \in C_{j} \subseteq C_{1} \Rightarrow H^{\prime} \in C_{|H|} \subseteq C_{j}$, and $C_{j} \backslash C_{k}$ is an antichain since $C_{j} \backslash C_{k} \subseteq C_{1} \backslash C_{|H|} \nsupseteq\left\{H^{\prime}, H^{\prime \prime}\right\}$.

We can conclude now the proof of Theorem 1 in the following three steps:

1. We use the structure of the compressed sets to find new expressions for the cardinalities of $B$ and $A+B$. Let $C_{1} \ldots C_{|H|}$ be as in Lemma 27. By interchanging the order of summation:

$$
|B|=\sum_{H^{\prime} \in G / H}\left|B \cap H^{\prime}\right|=\sum_{H^{\prime} \in G / H} \#\left\{j \in \mathbb{N}|| B \cap H^{\prime} \mid \geq j\right\}=\sum_{j=1}^{|H|}\left|C_{j}\right|
$$

We estimate $|A+B|$ in a similar fashion. For $1 \leq j \leq|H|$, suppose $H^{\prime \prime} \in \delta\left(C_{j}\right) \cup\{H\}$. We show that $A+B$ intersects $H^{\prime \prime}$ in $j$ elements at the least: 
- If $H^{\prime \prime}=H$, use $H \subseteq A$ and $0 \in B \neq \varnothing$ to obtain $\left|(A+B) \cap H^{\prime \prime}\right| \geq|(H+0) \cap H| \geq j$.

- Otherwise $H^{\prime \prime}=e_{h+i}+H^{\prime}$ for some $H^{\prime} \in C_{j}$ and $1 \leq i \leq m=\operatorname{codim} H$. Since $e_{h+i} \in E \subseteq A$, clearly $\left|(A+B) \cap H^{\prime \prime}\right| \geq\left|\left(e_{h+i}+B\right) \cap\left(e_{h+i}+H^{\prime}\right)\right|=\left|B \cap H^{\prime}\right| \geq j$.

Consequently:

$$
|A+B|=\sum_{H^{\prime \prime} \in G / H} \#\left\{j \in \mathbb{N}||(A+B) \cap H^{\prime \prime} \mid \geq j\right\} \geq \sum_{j=1}^{|H|}\left|\delta\left(C_{j}\right) \cup\{H\}\right|
$$

2. We use the isoperimetric inequality in order to obtain a lower bound on $|A+B|$ given $m$ and $|B|$. Let $0 \leq k \leq m$ and $w \in[-1,1]$ be such that:

$$
|B|=\frac{\left(\begin{array}{c}
m+1 \\
0
\end{array}\right)+\left(\begin{array}{c}
m+1 \\
1
\end{array}\right)+\ldots+\left(\begin{array}{c}
m+1 \\
k
\end{array}\right)+w\left(\begin{array}{c}
m \\
k
\end{array}\right)}{2^{m+1}} \cdot|G|
$$

We substitute $|B|=\sum\left|C_{j}\right|$ in the left-hand side, apply Pascal's rule to $\left(\begin{array}{c}m+1 \\ 1\end{array}\right) \ldots\left(\begin{array}{c}m+1 \\ k\end{array}\right)$ on the right-hand side, and divide both by $|H|=|G| / 2^{m}$, to obtain:

$$
\mathrm{E}[C]=\frac{1}{|H|} \sum_{j=1}^{|H|}\left|C_{j}\right|=\frac{|B|}{|H|}=\left(\begin{array}{c}
m \\
0
\end{array}\right)+\left(\begin{array}{c}
m \\
1
\end{array}\right)+\ldots+\left(\begin{array}{c}
m \\
k-1
\end{array}\right)+\frac{1+w}{2}\left(\begin{array}{c}
m \\
k
\end{array}\right)
$$

Now by Proposition 25

$$
\mathrm{E}[\{H\} \cup \delta C] \geq 1+\left(\begin{array}{c}
m \\
1
\end{array}\right)+\left(\begin{array}{c}
m \\
2
\end{array}\right)+\ldots+\left(\begin{array}{c}
m \\
k
\end{array}\right)+\frac{1+w}{2}\left(\begin{array}{c}
m \\
k+1
\end{array}\right)
$$

where the union is disjoint since always $H \notin \delta C_{j}$. In terms of $A$ and $B$, this implies:

$$
|A+B| \geq \frac{\left(\begin{array}{c}
m+1 \\
0
\end{array}\right)+\left(\begin{array}{c}
m+1 \\
1
\end{array}\right)+\ldots+\left(\begin{array}{c}
m+1 \\
k
\end{array}\right)+\left(\begin{array}{c}
m+1 \\
k+1
\end{array}\right)+w\left(\begin{array}{c}
m \\
k+1
\end{array}\right)}{2^{m+1}} \cdot|G|
$$

3. What values can $m=\operatorname{codim} H$ take? By Lemma $7(7),\left(1+\frac{m}{2}\right) / 2^{m} \geq|A| /|G|$, where the case $m=1$ is separately deduced from the assumption $|A| /|G| \leq 3 / 4$. On the other hand, by the theorem's assumption on $t,|A| /|G|>(t+2) / 2^{t+1}=\left(1+\frac{t}{2}\right) / 2^{t}$. Since the sequence $\left(1+\frac{n}{2}\right) / 2^{n}$ is monotone, we infer $m<t$.

The theorem is obtained by plugging $m=t-1$ into the derived lower bound. We claim that for smaller $m$ the bound is even higher, as demonstrated in Figure 3 . Indeed, for each $1 \leq i \leq t-2$, the graph of the lower bound on $|A+B|$ given $m=i$ is concave down by the log-concavity of the binomial coefficients, $\left(\begin{array}{c}m \\ k\end{array}\right) /\left(\begin{array}{c}m \\ k-1\end{array}\right) \geq\left(\begin{array}{c}m \\ k+1\end{array}\right) /\left(\begin{array}{c}m \\ k\end{array}\right)$. Thus the graph of $m=i+1$, which connects the midpoints of adjacent segments in the $m=i$ graph, must be lower.

Remark. By the Hamming balls construction, the lower bound we have found is optimal on a biparametric discrete family of points. In view of our treatment of $F(K)$ in the previous section, we expect that at intermediate points better bounds should be provable.

There are three points where our approach to Theorem 1 may be suboptimal: the isoperimetric inequality we use is not always perfectly tight, the addition of $H \cup E$ instead of the whole of $A$, and dropping the assumption on $B$ 's affine span.

It is perhaps worth remarking that the machinery of compressions can still be applied under the assumption $\langle A\rangle=\langle B\rangle=G$. This is done by showing that without loss of generality we may assume that $A$ and $B$ are simultaneously compressed such that they include a common affine basis.

Here is a brief outline of how this is done. First, partition $A$ and $B$ into their intersections with cosets of $\langle(A-A) \cap(B-B)\rangle$. These parts can be translated without increasing $|A+B|$, such that $A-A$ and $B-B$ include a common basis of $G$. Then apply $\{i\}$-compressions with respect to this basis, until it is included in $A \cap B$. 


\section{Acknowledgment}

I would like to thank my advisor, Professor Nati Linial, for his patient and helpful guidance during the research and the preparation of this manuscript.

\section{References}

[1] B Bollobás and I Leader. Sums in the grid. Discrete Mathematics, 162(1-3):31-48, 1996.

[2] A L Cauchy. Recherches sur les nombres. J. École Polytechnique, 9:99-123, 1813.

[3] G Cohen and G Zémor. Subset sums and coding theory, structure theory of set addition. Astérisque, 258, 1999.

[4] H Davenport. On the addition of residue classes. Journal of the London Mathematical Society, 1(1):30, 1935.

[5] J M Deshouillers, F Hennecart, and A Plagne. On small sumsets in $(\mathbb{Z} / 2 \mathbb{Z})^{n}$. Combinatorica, 24(1):53-68, 2004.

[6] H Diao. Freiman-Ruzsa-type theory for small doubling constant. Mathematical Proceedings of the Cambridge Philosophical Society, 146(2):269-276, 2009.

[7] S Eliahou and M Kervaire. Sumsets in vector spaces over finite fields. Journal of Number Theory, 71(1):12-39, 1998.

[8] S Eliahou and M Kervaire. Minimal sumsets in infinite abelian groups. Journal of Algebra, 287(2):449-457, 2005.

[9] S Eliahou and M Kervaire. Old and new formulas for the Hopf-Stiefel and related functions. Expositiones Mathematicae, 23(2):127-145, 2005.

[10] S Eliahou, M Kervaire, and A Plagne. Optimally small sumsets in finite abelian groups. Journal of Number Theory, 101(2):338-348, 2003.

[11] P Frankl. A new short proof for the Kruskal-Katona theorem. Discrete Mathematics, 48(2-3):327-329, 1984.

[12] P Frankl. The shifting technique in extremal set theory. Surveys in combinatorics, 123:81-110, 1987.

[13] P Frankl. A lower bound on the size of a complex generated by an antichain. Discrete mathematics, 76(1):51-56, 1989.

[14] G A Freiman. Foundations of a structural theory of set addition (translated from the Russian). Translations of Mathematical Monographs, 37, 1973.

[15] R J Gardner and P Gronchi. A Brunn-Minkowski inequality for the integer lattice. Transactions - American Mathematical Society, 353(10):3995-4024, 2001.

[16] B Green and I Z Ruzsa. Sets with small sumset and rectification. Bulletin of the London Mathematical Society, $38(1): 43,2006$.

[17] B Green and T Tao. Freiman's theorem in finite fields via extremal set theory. Combinatorics, Probability and Computing, 18(03):335-355, 2009.

[18] L H Harper. Optimal numberings and isoperimetric problems on graphs. Journal of Combinatorial Theory, 1(3):385-393, 1966.

[19] F Hennecart and A Plagne. On the subgroup generated by a small doubling binary set. European Journal of Combinatorics, 24(1):5-14, 2003.

[20] H Hopf. Ein toplogischer Beitrag zur reellen Algebra. Commentarii Mathematici Helvetici, 13(1):219-239, 1940.

[21] G O H Katona. Intersection theorems for systems of finite sets. Acta Mathematica Hungarica, 15(3):329-337, 1964.

[22] G O H Katona. The hamming-sphere has minimum boundary. Studia Sci. Math. Hungar, 10(1-2):131-140, 1975.

[23] S V Konyagin. On the Freiman theorem in finite fields. Mathematical Notes, 84(3):435-438, 2008.

[24] J B Kruskal. The number of simplices in a complex. Mathematical optimization techniques, page 251, 1963.

[25] V F Lev. Generating binary spaces. Journal of Combinatorial Theory, Series A, 102(1):94-109, 2003.

[26] V F Lev. Critical pairs in abelian groups and Kemperman's structure theorem. Int. J. Number Theory, 2(3):379-396, 2006.

[27] A Pfjster. Zur Darstellung von-1 als Summe von Quadraten in einem Körper. Journal of the London Mathematical Society, 1(1):159, 1965.

[28] I Z Ruzsa. Arithmetical progressions and the number of sums. Periodica Mathematica Hungarica, 25(1):105-111, 1992.

[29] I Z Ruzsa. Generalized arithmetical progressions and sumsets. Acta Mathematica Hungarica, 65(4):379-388, 1994.

[30] I Z Ruzsa. Sum of sets in several dimensions. Combinatorica, 14(4):485-490, 1994.

[31] I Z Ruzsa. An analog of Freiman's theorem in groups. Astérisque, pages 323-326, 1999. 
[32] T Sanders. A note on Freiman's theorem in vector spaces. Combinatorics, Probability and Computing, 17(02):297-305, 2008.

[33] R Schneider. Convex bodies: the Brunn-Minkowski theory, volume 44. Cambridge Univ Pr, 1993.

[34] D B Shapiro. Compositions of quadratic forms, volume 33. Walter De Gruyter Inc, 2000.

[35] E Stiefel. Über Richtungsfelder in den projektiven Räumen und einen Satz aus der reellen Algebra. Commentarii Mathematici Helvetici, 13(1):201-218, 1940.

[36] T Tao and V Vu. Additive combinatorics. Cambridge Studies in Advanced Mathematics, $105,2006$.

[37] E Viola. Selected results in additive combinatorics: An exposition. Electronic Colloquium on Computational Complexity (ECCC), 14(103), 2007.

[38] A G Vosper. The critical pairs of subsets of a group of prime order. Journal of the London Mathematical Society, 1(2):200, 1956.

[39] S Yuzvinsky. Orthogonal pairings of euclidean spaces. The Michigan Mathematical Journal, 28(2):131-145, 1981.

[40] G Zémor. An extremal problem related to the covering radius of binary codes. Algebraic Coding, pages 42-51, 1992.

[41] G Zémor. Subset sums in binary spaces. European journal of combinatorics, 13(3):221-230, 1992. 